\title{
Single synapses control mossy cell firing
}

Alexander Drakew ${ }^{1^{*}, \bowtie}$, Urban Maier $^{1^{*}}$, Anja Tippmann $^{1,2}$, Michael Frotscher $^{1 \dagger}$

${ }^{1}$ Institute for Structural Neurobiology, Centre for Molecular Neurobiology Hamburg (ZMNH), University Medical Centre Hamburg-Eppendorf, 20251 Hamburg, Germany

${ }^{2}$ current address: Systems Neuroscience, Johann-Friedrich-Blumenbach Institute for Zoology and Anthropology, Georg August University Göttingen, 37075 Göttingen, Germany

*both authors contributed equally to this study

${ }^{\circledR}$ Correspondence to: A.D. (alexander.drakew@zmnh.uni-hamburg.de)

†Michael Frotscher passed away on May, 272017 


\begin{abstract}
The function of mossy cells (MCs) in the dentate gyrus has remained elusive. Here we determined the functional impact of single mossy fibre (MF) synapses on MC firing in mouse entorhino-hippocampal slice cultures. We stimulated single MF boutons and recorded $\mathrm{Ca}^{2+}$ transients in the postsynaptic spine and unitary excitatory postsynaptic potentials (EPSPs) at the MC soma. Synaptic responses to single presynaptic stimuli varied strongly between different MF synapses, even if they were located on the same MC dendrite. Synaptic strengths ranged from subthreshold EPSPs to direct postsynaptic action potential (AP) generation. Induction of synaptic plasticity at these individual MF synapses resulted in potentiation or depression depending on the initially encountered synaptic state, indicating that synaptic transmission at MF synapses on MCs is determined by their previous functional history. With these unique functional properties MF-MC synapses control MC firing individually thereby enabling modulation of the dentate network by single granule cells.
\end{abstract}


Sensory input that reaches the entorhinal cortex from visual, olfactory and auditory centres is relayed to the hippocampus via distinct connectivity channels formed by the synapses of dentate granule cells ${ }^{1}$. The mossy fibres (MFs), the axons of the granule cells, give rise to giant presynaptic boutons that terminate on large complex spines on the proximal dendritic segments of pyramidal neurons ${ }^{2-5}$ and on dendritic shafts of GABAergic interneurons $^{6,7}$ in the CA3 region. However, on their way to CA3 MFs first encounter the mossy cells (MCs) in the hilus of the dentate gyrus ${ }^{8}$. In contrast to CA3 pyramidal neurons, MCs feature MF synapses on all dendritic segments and on various types of spines ${ }^{9}$. MF-MC synapses are thought to be equivalent to MF-CA3 synapses in showing presynaptic expression of LTP and frequency facilitation ${ }^{10,11}$. Being embedded in the hilus and exposed to abundant bypassing MFs, single MCs may receive input from several hundred (800) granule cells ${ }^{12}$. Thus, the specific segregated granule cell output, which conveys information about contextual changes in the external environment from the entorhinal cortex to the hippocampus, converges on $\mathrm{MCs}^{13-16}$.

MCs are excitatory glutamatergic neurons ${ }^{17}$ that project to the hilus locally and to the inner molecular layer of distant portions of the ipsilateral and contralateral dentate gyrus $^{18,19}$, innervating numerous granule cells and dentate inhibitory interneurons in both hemispheres. MCs are thought to modulate the excitation/inhibition balance of dentate granule cells differentially in local and distant parts of the dentate gyrus thereby promoting the formation of functional transversal lamellae in the hippocampus $8,20,21$. Consistent with this idea, genetic ablation of MCs resulted in transient granule cell hyperexcitability and impaired pattern separation ${ }^{22}$. Furthermore, MCs are the first excitatory neurons that activate newly generated granule cells, which extend their yet short dendrites to the inner molecular layer ${ }^{23}$. Comparative studies revealed no principal differences between MCs in rodents and primates ${ }^{9}$, suggesting that $\mathrm{MCs}$ are an important, conserved component of the hippocampal network. 
A remarkable feature of MF synapses is their structural plasticity. Structural complexity of MF boutons was found to increase with the age of the animal and in response to enriched environment ${ }^{24}$. Similarly, in hippocampal slice cultures the complexity of MF boutons increased with the incubation period in vitro and with functional activity, thus establishing slice cultures as a tool to study MF synaptic plasticity ${ }^{24,25}$. In line with these results, stimulation of single granule cells increased the complexity of their respective MF boutons ${ }^{26}$. Earlier studies had already shown that the number and size of complex spines postsynaptic to MF boutons were reduced following removal of the entorhinal cortex ${ }^{27}$. Collectively these results suggest that MF synapses change their structural components individually depending on the activity of the parent granule cell in response to environmental stimuli mediated by the entorhinal cortex.

While these morphological studies revealed structural variability of MF synapses, they could not clarify whether individual MF synapses were functionally different. Functional synaptic modification, such as potentiation of synaptic transmission ${ }^{28}$ is assumed to underlie adaptive mechanisms such as learning and memory ${ }^{29,30}$ and is likely to occur at individual synapses depending on their previous individual involvement in active microcircuits. Hence, at a given point in time one would expect to envisage different, non-random states of synaptic transmission in a defined population of synapses, such as MF synapses. Moreover, little is known about the detonation properties, the efficacy, and plasticity of individual MF-MC synapses.

In the present study, we hypothesized that the synaptic strengths of individual MF synapses on MCs are heterogeneous and are modified individually by activity. We tested the functional behaviour of individual synapses by activating single MF boutons on $\mathrm{MC}$ dendrites, recording $\mathrm{Ca}^{2+}$ transients in spines postsynaptic to these boutons and unitary EPSPs at the MC soma, and inducing plasticity at these synapses using a defined stimulation protocol. 


\section{Results}

\section{Determining the synaptic states of individual MF synapses on MCs}

We chose organotypic entorhino-hippocampal slice cultures as a model system to study the individual states of single synapses under stable in vitro conditions. Acute slices would have been an alternative model for studies of that kind. However, slice preparation has been shown to change acutely the number of spines and synapses in response to acute deafferentation by the transection of many axons and dendrites ${ }^{31}$. Thus, states of synaptic transmission may not reflect previous synaptic activity under these conditions. We used organotypic entorhino-hippocampal slice cultures that were incubated in vitro for at least 4 weeks to allow for recovery and stabilization of the tissue following slice preparation, the removal of debris, and for the maturation of the MF projection. Such long-term slice cultures were shown before to represent a reliable model for the study of MF synapses since the development and activity-induced structural plasticity of MF synapses were very similar in vivo and in slice culture ${ }^{24}$. In the present study we used hippocampal slice cultures with the entorhinal cortex attached to preserve major afferent projections to the granule cells which retain their layer-specific terminations under these in vitro conditions ${ }^{32}$. Moreover, the fine-structural characteristics of MF synapses were preserved in slice culture and there were no principal differences to MF synapses in vivo ${ }^{33}$. Accordingly, this in vitro model has been successfully used in studies on the functional and structural plasticity of MF synapses 24,34 .

MCs were patch-clamped and filled with Alexa 594 dextran for cell identification (Fig. 1a, d) and with Fluo-4FF for two-photon $\mathrm{Ca}^{2+}$ imaging (Fig. 1b, see Methods). Targeted patch-clamp recording of MF boutons contacting the labelled spines of the MC dendrite was facilitated by transiently staining the extracellular space with Alexa 488 hydrazide, which was not taken up by cellular elements, allowing to visualise the boutons 
as "shadows" surrounding the $\operatorname{spines}^{35}$ (Fig. 1b, c). Stimulation of MF boutons in looseseal cell-attached mode was combined with recording of unitary EPSPs from the target MC soma and simultaneous two-photon imaging of $\mathrm{Ca}^{2+}$ transients in the postsynaptic spine (Fig. 1e, f, Supplementary Fig. 1a-c, Supplementary Table 1). EPSPs following bouton stimulation were indistinguishable from EPSPs in response to spontaneous input to the same cell (Supplementary Fig. 2a). $\mathrm{Ca}^{2+}$ transients observed in the imaged spines reliably reported synaptic events occurring at this particular synapse since suprathreshold EPSPs generated somewhere else in the dendritic tree elicited only very small $\mathrm{Ca}^{2+}$ transients in the spine when compared to local EPSPs (Supplementary Fig. 2b, c).

We used paired-pulse stimulation of the MF bouton to interrogate individual MF synapses since the response to the first of the two stimuli allowed us to capture the current state of the synapse, while the voltage response to the second stimulus reported presynaptic facilitation known to increase transmitter release probability at MF synapses ${ }^{36}$. Paired-pulse facilitation, expressed as ratio of the second to the first response (pairedpulse ratio), allowed us to probe the initial release probability ${ }^{37}$. Paired-pulse facilitation is a form of short-term synaptic plasticity that needs to be differentiated from long-term changes of synaptic strength. Accordingly, presynaptic facilitation did not accumulate with repetitive paired-pulse stimulations in the synapses we studied. However, the non-invasive loose-seal bouton-attached configuration turned out to be stable enabling us to probe transmission at the same synapse again following induction of long-term synaptic potentiation applying an associative protocol ${ }^{38}$. This approach allowed us to analyse simultaneously presynaptic and postsynaptic components of transmission before and after induction of synaptic plasticity at single identified synapses. Of note, these experiments were performed in ACSF without any pharmacological additives at near physiological temperature. 


\section{MF synapses on MCs display heterogeneous synaptic states}

MF synapses on CA3 pyramidal cells are "conditional detonators", discharging the postsynaptic cell only in response to a train of granule cell APs ${ }^{39-41}$. In contrast, in $85 \%$ of the MF synapses on MCs we observed direct AP firing of the MC ("direct detonation") in response to the first stimulus (Fig. 1f, Fig. 2a, b, Supplementary Fig. 3).

Differences in synaptic states between individual MF synapses on MCs might be due to differences between slice cultures or MCs. Therefore, we early on made attempts to stimulate and record two MF synapses on the same MC. One of these experiments $(n=3)$ is shown in Fig. 1d-f. When we stimulated two different MF boutons contacting different spines on the same MC dendrite we noticed great differences in unitary EPSPS and postsynaptic $\mathrm{Ca}^{2+}$ transients between the two synapses on the same neuron. While stimulation of the synapse on spine 1 resulted only in subthreshold EPSPs, accompanied by $\mathrm{Ca}^{2+}$ transients of low amplitude, stimulation of the synapse on spine 2 led to frequent postsynaptic APs and large $\mathrm{Ca}^{2+}$ transients in the spine. Similarly, paired-pulse facilitation was different between the two synapses. These differences between the two synapses imply that the granule cell input to spine 2 , but not that to spine 1 , would in vivo be conveyed by the MC axon to large dorso-ventral portions of the ipsi- and contralateral dentate gyrus. We accordingly classified postsynaptic responses to bouton stimulation as subthreshold and suprathreshold, respectively, taking the different impact of a detonating synapse and a subthreshold synapse on network activity into account. MF synapses were thus of three response categories following the first stimulus: only subthreshold EPSPs, mixed sub- and suprathreshold responses, and only suprathreshold EPSPs (Fig. 2a, b, Supplementary Fig. 3). The fraction of suprathreshold responses of each synapse was positively correlated with the median EPSP slope but neither of these parameters was correlated with the dendritic distance of the synapse from the MC soma, indicating that 
the detonator status does not merely reflect proximity to the cell body (Supplementary Figs. 3 and $4 a-h)$.

Responses to the first stimulus revealed a great variability of the EPSP slope and peak $\mathrm{Ca}^{2+}$ amplitude in the total of 26 synapses studied in 26 slice cultures, each colourcoded individually allowing for their identification in the various figures (Fig. $2 b$, Supplementary Fig. 3). Despite the observed variability, EPSP slope and peak $\mathrm{Ca}^{2+}$ response were positively correlated within single synapses (statistically significant correlation in 22 of 26 synapses, Supplementary Fig. 5) and among synapses (Fig. 2b). Of note, the different synaptic weights in response to repeated paired-pulse stimulations of a synapse were not correlated with the sequential order of repetitions, precluding accumulation of synaptic facilitation (no significant correlation of sequential order with EPSP slopes in 23 of 26 synapses and with peak $\mathrm{Ca}^{2+}$ in 21 of 26 synapses, Supplementary Fig. 5). The regression lines of EPSP slopes and peak $\mathrm{Ca}^{2+}$ responses differed remarkably between individual synapses, as did their amplitudes (Fig. 2a, b, Supplementary Fig. 3), pointing to variable glutamate release and variable AMPA and NMDA glutamate receptor contributions. Consistent with this hypothesis, varying the amount of glutamate in two-photon uncaging experiments resulted in corresponding changes in voltage responses (uncPSPs) and $\mathrm{Ca}^{2+}$ responses (Fig. 3a-c). Comparable to single bouton stimulation, we observed a great heterogeneity of the responses from all 79 synapses studied in these uncaging experiments (Fig. 3d, e). Application of the AMPA receptor antagonist CNQX almost completely abolished the uncPSP amplitudes, whereas treatment with the NMDA receptor blocker D-APV resulted in a drastic reduction of peak $\mathrm{Ca}^{2+}$ responses (Fig. 3f, g), indicating that EPSP amplitudes represent AMPA receptor activation and $\mathrm{Ca}^{2+}$ transients NMDA receptor currents at MF synapses similar to other synapses $^{42-44}$. In contrast to single bouton stimulation, we did not observe suprathreshold 
responses to glutamate uncaging, reflecting that glutamate uncaging involved only a subset of postsynaptic densities contacted by a single MF terminal.

Presynaptic facilitation assessed by the paired-pulse ratio of EPSP slope revealed a contribution of the bouton to synaptic heterogeneity showing a strong facilitation of primarily weak synapses and weak facilitation or depression of strong synapses. Weak responses to the first stimulus were correlated with strong paired-pulse ratios of EPSP slope among synapses as well as within individual synapses, indicating that the variability of the first synaptic response is due to a variable initial release probability (Fig. 2c, Supplementary Figs. 3 and 6). Suprathreshold EPSPs following both stimuli occurred on average in $57 \%$ of all stimulations per synapse, whereas only one or no AP at all was observed in $23 \%$ and $19 \%$, respectively (Table 1). Taken together, single bouton stimulation disclosed a great deal of heterogeneity of EPSP slopes and peak $\mathrm{Ca}^{2+}$ responses, pointing to individual contributions of single MF synapses to MC firing ranging from consistently subthreshold activation to robust detonation.

\section{Divergent plasticity following a uniform induction protocol}

What determined the observed heterogeneity of MF synapses? The response to the first of paired stimuli represents the synaptic state at this particular point in time. Different strengths of synapses may indicate different histories of potentiation and depression. This would imply that the state of an individual MF synapse is the result of previous activity and is further modified by ongoing activity. Therefore, following the paired-pulse protocol, we induced long-term synaptic plasticity at these same synapses by a single train of 24 bouton stimulations $(25 \mathrm{~Hz})$, each paired with a delayed (+ $10 \mathrm{~ms}$ ) backpropagating action potential (bAP) elicited in the postsynaptic cell (spike-timing-dependent plasticity ${ }^{38}$ ) (Fig. 4a-e). Our analysis of responses to paired-pulse stimulation, reporting changes in release probability and changes in EPSP slope and $\mathrm{Ca}^{2+}$ transients, allowed us to 
determine both presynaptic and postsynaptic plasticity (Fig. 5, Supplementary Fig. 7, Table 2). We noticed great differences in the changes of synaptic strengths and of paired pulse ratios 30 minutes after the combined stimulation (Fig 5a, b, Supplementary Fig. 7). Previously weak synapses showing initially small EPSP slopes, low peak $\mathrm{Ca}^{2+}$ amplitudes, and none or only a small fraction of suprathreshold EPSPs in response to the first of paired stimuli became potentiated, whereas previously strong synapses remained strong or became even depotentiated (Fig. 5c-e, Supplementary Fig. 7). However, all but one synapse showed an increase or no change in the fraction of suprathreshold EPSPS in response to the first of paired stimuli: Potentiation of 5 out of 11 synapses resulted in a change of the detonator status to the mixed sub- and suprathreshold and the all suprathreshold response category, respectively (Fig. 5f, Supplementary Fig. 7). Considering both responses to paired-pulse stimulation, the fraction of suprathresholdonly responses to both stimuli had increased significantly $30 \mathrm{~min}$ after induction of plasticity, whereas the fraction of paired-pulse responses containing at least one subthreshold EPSP decreased accordingly (Table 2). The paired-pulse ratio of EPSP slope decreased in most of the potentiated synapses. The synapses with the largest increase in EPSP slope showed the strongest reduction in paired-pulse ratio indicating that potentiation of these synapses was due to an increase in presynaptic release probability (Fig. 5b, Supplementary Fig. 7). Overall, these findings indicate that the outcome of plasticity depended on the initially encountered synaptic state of these MF synapses. Vice versa, our findings suggest that the initially encountered synaptic state had resulted from the different previous activities of these individual MF synapses. 


\section{Discussion}

In the present study, we provide evidence for a dynamic control of MC firing by single MF inputs that are separately regulated by state-dependent individual synaptic plasticity. Synaptic potentiation using an associative protocol was found to change synaptic strengths differentially, depending on the initially encountered state of the synapse. We probed the initial synaptic weight by monitoring the first response to paired-pulse stimulation and compared the response to the first stimulus before and 30 minutes after induction of synaptic plasticity. While this approach did not allow us to trace the history of the synaptic state back to before initial stimulation, we were able to follow the plastic response to a defined stimulus train, which was different in the synapses studied leading to potentiation but also depression. This bidirectional plasticity was observed pre- and postsynaptically and is consistent with the multitude of plasticity mechanisms reported for MF synapses $36,45-48$.

We simultaneously measured peak $\mathrm{Ca}^{2+}$ amplitudes and EPSP slopes in response to single MF bouton stimulations. These two parameters were correlated in individual synapses, but each synapse displayed a distinct relation between $\mathrm{Ca}^{2+}$ and unitary voltage responses (Fig. 2a, b, Supplementary Fig. 3). A likely explanation is that some MF synapses on MCs have many NMDA receptors while others have only very few. This is supported by glutamate-uncaging experiments, where we also found heterogeneity in $\mathrm{Ca}^{2+}$ to voltage relation, showing that this heterogeneity is due to postsynaptic mechanisms (Fig. 3d, e). Moreover, the uncaging experiments revealed that NMDA receptor blockade dramatically reduced $\mathrm{Ca}^{2+}$ transients, whereas blockade of AMPA receptors strongly decreased uncPSP amplitudes (Fig. 3f, g). Since glutamate uncaging was restricted to single spine heads, the heterogeneity in the ratio of peak $\mathrm{Ca}^{2+}$ amplitudes to EPSP slopes of synaptic responses could not be attributed merely to a potentially 
varying number of spine heads activated by a single MF bouton. Within single synapses EPSP slope was negatively correlated with paired-pulse ratio indicating that the observed heterogeneity of responses to the first pulse was based on a strong variability of release probability within single boutons (Supplementary Figs. 3 and 6). Between synapses we also found a pronounced heterogeneity of release probability as indicated by the pairedpulse ratios of EPSP slopes ranging from strong facilitation to depression. Thus, we encountered also the MF boutons in varying states corresponding to the postsynaptic heterogeneity (Fig. 2c).

Induction of plasticity at these synapses using an associative protocol resulted in correlated changes in peak $\mathrm{Ca}^{2+}$ amplitudes and EPSP slopes and corresponding changes in paired-pulse ratios even though some synapses were strengthened and others became weakened, pointing to a change in presynaptic glutamate release probability (Fig. 5). However, in 5 out of 11 synapses we observed pronounced changes in the $\mathrm{Ca}^{2+}$ to voltage relation, suggesting additional postsynaptic modifications (Supplementary Fig. 7). The direction of plasticity was not random, but depended on the initial state of the synapse, since weak synapses tended to be potentiated whereas already strong synapses showed depotentiation (Fig. 5c-e).

Plasticity at MF synapses has been shown to be expressed presynaptically, modulating glutamate release probability and resulting in potentiation ${ }^{36}$ or depotentiation ${ }^{49}$. Presynaptic GABAergic ${ }^{50}$, cholinergic ${ }^{51}$ and glutamatergic mechanisms are likely to contribute to MF bouton plasticity. More recent studies have revealed additional postsynaptic forms of plasticity at MF synapses, including NMDA receptor-dependent recruitment of NMDA receptors ${ }^{46,52}$, NMDA receptor-dependent potentiation of AMPA excitatory postsynaptic currents ${ }^{47}$, and NMDA receptor-dependent bidirectional spiketiming-dependent plasticity ${ }^{53}$. Depression of postsynaptic NMDA currents may result from the co-release of zinc from strongly activated MF boutons ${ }^{54}$. Given the heterogeneity in 
glutamate receptor content of MF-MC synapses reported here, one would expect heterogeneous responses to a defined stimulus, as was indeed observed when applying a defined plasticity protocol. Different forms of synaptic plasticity are generally attributed to different stimulation protocols. In MF-MC synapses we found that pre- and postsynaptic plasticity depended on the initial state of the synapse. At least for MF synapses on MCs, we have shown that there is a great diversity of synaptic states. Apart from the detonation property, which is a binary response variable, we found a continuum of synaptic states rather than discrete modes of transmission as suggested for synapses between CA3 pyramidal cells ${ }^{55}$. We observed plasticity of voltage and $\mathrm{Ca}^{2+}$ responses following a uniform protocol, spanning a wide range of changes without obvious clustering of response patterns, thus not supporting a shift between discrete modes of MF-MC synapses.

We conclude that the different behaviour of individual MF synapses is based on their individual functional history, but other factors may also contribute. The dendritic distance between the synapses and the soma varied to some extent although neither the fraction of suprathreshold responses nor the median slopes of the EPSPs did correlate with this distance (Supplementary Figs. 3 and 4). MF synapses are heterogeneous with respect to their structural components such as bouton size, the complexity of postsynaptic spines and the number of active zones ${ }^{4,5}$. However, increased structural complexity of MF synapses was found to result from their previous activity ${ }^{24}$. Thus, structural changes of individual synapses are likely to contribute to the observed functional heterogeneity of MF synapses on MCs.

We noticed different synaptic states with respect to postsynaptic MC firing in response to the first stimulus, ranging from subthreshold-only responses in one group of synapses to suprathreshold-only responses in another (Table 1, Fig. 2, Supplementary Fig. 3). While a suprathreshold MF synapse on a MC may eventually result in the activation 
of large populations of granule cells and interneurons in distant lamellae in the ipsi- and contralateral hippocampus, these same neurons will not be activated by a subthreshold MF-MC synapse (Fig. 6a, b). Fifteen out of the 26 synapses show both suprathreshold and subthreshold responses to the first of paired stimuli. One is tempted to speculate that these mixed synapses are toggling between sub- and suprathreshold responses due to the analogue control of release probability by the integration of presynaptic granule cell EPSPs in MF boutons ${ }^{56}$. Thus, MF-MC synapses might function as a metastable network switch, depending on the granule cell firing rate as well as the strength of a granule cell EPSP accompanying the presynaptic $\mathrm{AP}^{56}$ (Fig. 6c). In response to an associative induction protocol many synapses showed an increased fraction of suprathreshold EPSPs, suggesting that synaptic activity changes the detonator status of MF-MC synapses (Table 2, Fig. 5f, Supplementary Fig. 7). We conclude that MF-MC synapses integrate previous activity to become stronger or weaker, thus controlling modulation of the dentate network by switching between detonating and non-detonating MC activation. The results imply that single granule cells control $\mathrm{MC}$ firing in a precisely regulated manner reflecting their synaptic input patterns. Direct detonation of MCs by single granule cells as shown here is well compatible with previous reports on the high firing rate of MCs in vivo when compared to the sparse firing of granule cells ${ }^{15,18,40}$.

Detonation of a MC by a single granule cell promotes di-synaptic inhibition of dentate granule cells locally and excitation as well as di-synaptic inhibition of granule cells in distant parts of the dentate gyrus ${ }^{8}$. A single bursting granule cell having established a full detonator synapse with a MC may potentiate the MCs output synapses thereby shifting the excitation/inhibition balance of large numbers of distant granule cells postsynaptic to that $\mathrm{MC}^{21}$. Recent optogenetic studies provided evidence for MCs being the first excitatory input to adult-generated granule cells when their growing dendrites reach the inner molecular layer, the termination zone of distal MC axons in the ipsi- and contralateral 
dentate gyrus ${ }^{23}$. Thus, the MC input may be important for the network integration of newly generated granule cells, which have been shown to mediate pattern separation, whereas old granule cells facilitate pattern completion ${ }^{57}$. Remarkably, genetic ablation of MCs resulted in impaired pattern separation ${ }^{22}$, suggesting that MCs controlled by the MF input synapses play a crucial role in the integration of new granule cells and contextual discrimination mediated by these neurons. The conditional detonator synapses of MFs on CA3 pyramidal cells are known to be involved in complex network functions such as pattern completion and pattern separation ${ }^{57-59}$. The metastable direct detonator MF-MC synapses introduced here may accordingly play a critical role in the functional organization of the dentate gyrus. 


\section{Methods}

\section{Preparation of organotypic slice cultures}

Mouse pups of both sexes (P4 to P5; C57bl6, local colony) were decapitated with scissors in accordance with institutional, European and international guidelines (licence no. ORG_582). The entorhino-hippocampal complex was dissected out of the brains and cut horizontally at $300 \mu \mathrm{m}$ thickness using a Mc Illwain tissue chopper. Slices containing the entorhinal cortex attached to the ventral hippocampus were transferred to Millicell inserts (Millipore, Schwalbach, Germany) ${ }^{60}$ and incubated for at least 28 days at $37^{\circ} \mathrm{C}$ and $5 \% \mathrm{CO}_{2}$ in $1.2 \mathrm{ml}$ of a medium composed of $50 \%$ Minimum Essential Medium, $25 \%$ Basal Medium Eagle, 25\% heat-inactivated horse serum, $2 \mathrm{mM}$ glutamine, and $6.5 \mathrm{mg} / \mathrm{ml}$ glucose with $\mathrm{pH}$ adjusted to 7.3. The medium was exchanged three times per week. Substances were obtained from Invitrogen (Darmstadt, Germany).

\section{Patch clamp recording solutions}

Slice cultures were superfused at $35^{\circ} \mathrm{C}$ (measured $1 \mathrm{~mm}$ beneath the slice) with artificial cerebrospinal fluid (ACSF) containing $125 \mathrm{mM} \mathrm{NaCl}, 25 \mathrm{mM} \mathrm{NaHCO}$, $2.5 \mathrm{mM} \mathrm{KCl}$, $1.25 \mathrm{mM} \mathrm{NaH}_{2} \mathrm{PO}_{4}, 1 \mathrm{mM} \mathrm{MgCl}, 25 \mathrm{mM}$ glucose, and $2 \mathrm{mM} \mathrm{CaCl}, \mathrm{pH}$ adjusted to 7.30 with $\mathrm{NaOH}, \sim 305$ mOsm, equilibrated with $95 \% \mathrm{O}_{2}$ and $5 \% \mathrm{CO}_{2}$.

The MNI-glutamate uncaging was performed in a self-contained volume of $0.7 \mathrm{ml}$ ACSF composed of $120 \mathrm{mM} \mathrm{NaCl}, 25 \mathrm{mM} \mathrm{NaHCO}_{3}, 2.5 \mathrm{mM} \mathrm{KCl}, 1.25 \mathrm{mM} \mathrm{NaH}_{2} \mathrm{PO}_{4}$, $1 \mathrm{mM} \mathrm{MgCl}_{2}, 10 \mathrm{mM}$ glucose, and $2 \mathrm{mM} \mathrm{CaCl}_{2}$, and $20 \mathrm{mM}$ 4-Methoxy-7-nitroindolinyl (MNI)-glutamate (Tocris, via BIOZOL, Eching, Germany). We needed a high concentration of MNI-glutamate for uncaging to keep the uncaging pulses short while 
minimizing laser intensities to mimic glutamate release kinetics without damaging the synapse. Moreover, multiquantal release at MF synapses ${ }^{61}$ requires uncaging of larger amounts of glutamate when compared to the small Schaffer collateral synapses in CA1. Glutamate receptor antagonists $(200 \mu \mathrm{M}$ 2-amino-5-phosphonopentanoic (D-APV) and $20 \mu \mathrm{M}$ 6-cyano-7-nitroquinoxaline-2,3-dione (CNQX) (both from ascent scientific, Cambridge, U.K.) were present in some uncaging experiments. The solution was covered by a constant flow of moistened gas $\left(95 \% \mathrm{O}_{2}\right.$ and $\left.5 \% \mathrm{CO}_{2}\right)$ and was heated locally to $\sim 35^{\circ} \mathrm{C}$. Evaporation was compensated for by continuous addition of water using a roller tube pump to keep osmolarity at 305 mOsm.

The pipette solution for whole-cell recordings contained $133 \mathrm{mM} \mathrm{K}$-gluconate, $12 \mathrm{mM} \mathrm{KCl}, 10 \mathrm{mM}$ HEPES, 7 mM Na -phosphocreatinine, 4 mM Mg-adenosine-5triphosphate, $0.3 \mathrm{mM}$ Na-guanosine-5-triphosphate, $9 \mathrm{mM}$ sucrose (to achieve $305 \mathrm{mOsm}$ ), $\mathrm{pH}$ adjusted to 7.30 with $\mathrm{KOH}$, and either $200 \mu \mathrm{M}$ Fluo-5F and $100 \mu \mathrm{M}$ Alexa-594 dextran (10 kD) for glutamate-uncaging experiments or $800 \mu \mathrm{M}$ Fluo-4FF and $40 \mu \mathrm{M}$ Alexa-594 dextran $(10 \mathrm{kD})$ for the rest of the experiments. Electrode tips were filled with intracellular solution not containing the dyes in order to avoid excessive background labelling of the tissue before seal formation. We used Alexa 594 dextran rather than the hydrazide form for intracellular labelling to avoid effects of the carbonyl-reactive hydrazide ${ }^{62}$ and used different $\mathrm{Ca}^{2+}$ indicators with different affinities since we expected larger $\mathrm{Ca}^{2+}$ transients in the bouton stimulation experiments ${ }^{63}$.

The pipette solution for loose-seal MF bouton stimulation contained $145 \mathrm{mM} \mathrm{NaCl}$, 2.5mM KCl, $1 \mathrm{mM} \mathrm{MgCl}_{2}, 2 \mathrm{mM} \mathrm{CaCl} 2,10 \mathrm{mM}$ HEPES, $\mathrm{pH}$ adjusted to 7.40 with $\mathrm{NaOH}$, and $50 \mu \mathrm{M}$ Alexa 488 hydrazide. Since dextran conjugates are actively taken up by neurons from external solutions, we used Alexa 488 hydrazide for transient labelling of the extracellular space for shadow patching. Albumin (Albumin bovine Fraction V, $25 \mathrm{mg} / \mathrm{ml}$, 
SERVA, Heidelberg, Germany) was added to reduce seal resistance. Chemicals were obtained from Sigma-Aldrich (Taufkirchen, Germany) and dyes from Invitrogen.

\section{Electrophysiology}

Mossy cells in the hilar region of the hippocampus were visualized under video IR-Dodt contrast $^{64}$, and whole-cell access was established with 5-10 $\mathrm{M} \Omega$ patch pipettes fabricated from borosilicate glass capillaries (outer diameter $2 \mathrm{~mm}$, inner diameter $1 \mathrm{~mm}$; Hilgenberg, Malsfeld, Germany) using a small piezo-driven micromanipulator (Kleindiek-nanotechnik, Reutlingen, Germany) for long-term stability of the recordings. On break in, resting membrane potentials ranged from $-63 \mathrm{mV}$ to $-78 \mathrm{mV}(-55 \mathrm{mV}$ to $-65 \mathrm{mV}$ in MNI-glutamate solution). Cells were recorded in current clamp mode using an ELC-03X amplifier (npi electronic, Tamm, Germany) in bridge mode. Series resistance and pipette capacitance compensation were adjusted using a phase-sensitive technique ${ }^{65}$. Amplifier output was low-pass Bessel-filtered at $20 \mathrm{kHz}$ and digitized at $100 \mathrm{kHz}$ using a PC-board (NI PCl6251, National Instruments, Munich, Germany) and in-house software based on LabVIEW $^{\mathrm{TM}}$ (National Instruments). The input resistance of MCs was determined by the ratios of the steady state voltage responses to small current steps (200 ms) ranging from -20 to +20 pA. Membrane time constant was derived from exponential fits of the voltage response to hyperpolarizing current steps (150 ms, -20 pA). AP firing threshold was defined independently of synaptic stimulation as the mean membrane potential right before the first elicited APs in response to depolarizing current steps of increasing amplitude (iterated until five steps with AP responses were obtained). Back-propagating action potentials (bAPs) were elicited by short somatic current injections (1.5 nA, $1.5 \mathrm{~ms}$ ).

Labelled spines of the patched mossy cells were approached using a second pipette (theta borosilicate glass, outer and inner diameter $2 \mathrm{~mm}$ and $1.4 \mathrm{~mm}$, respectively; 
Hilgenberg) filled with Alexa 488 hydrazide in the extracellular solution. Presynaptic MF boutons were identified as shadows covering labelled spines, contrasting against the stained extracellular space ${ }^{35}$, and loose-seal cell-attached mode was established ${ }^{66,67}$. MF boutons were stimulated with $0.5 \mathrm{~ms}$ pulses using a second ELC-03X amplifier (npi electronic) in current clamp x 10 mode (median: $36.6 \mathrm{nA}$, range 15 to $75 \mathrm{nA}$ ). Adequate stimulation intensity was determined for each bouton by increasing the current amplitude until an EPSP was recorded reliably in the postsynaptic cell with a maximal delay of $1.0 \mathrm{~ms}$ following the end of the stimulus (mean delay: $0.546 \mathrm{~ms} \pm 0.188$ s.d.; 26 synapses). Voltage and $\mathrm{Ca}^{2+}$ responses were all-or-none with amplitudes independent of the bouton stimulation intensity (Supplementary Fig. 1a-c). Due to the large stimulus artefact recorded in the presynaptic pipette we were not able to monitor the AP elicited in the bouton in loose-seal cell-attached mode. Therefore, we did not attempt to determine failure rates using stimulation at threshold. During presynaptic stimulation a very small depolarization of the postsynaptic cell by less than $0.2 \mathrm{mV}$ was recorded that typically returned back to the pre-stimulus potential before the onset of the EPSP, indicating synaptic activation rather than direct stimulation of the postsynaptic cell (Supplementary Fig. 1a-c). Moreover, contamination of the recorded EPSPs with synaptic responses following antidromic AP propagation to the same MC is unlikely, given the spacing of MF boutons ${ }^{68}$ and a propagation speed of $\sim 210 \mu \mathrm{m} / \mathrm{ms}^{69}$. Indeed, we did not observe abrupt increases of the voltage slope during the rising phase of the EPSPs that would have been indicative of such compound signals.

Paired-pulse stimuli with an inter-stimulus interval of $40 \mathrm{~ms}$ were applied to test pre- as well as postsynaptic components of synaptic transmission. Paired-pulse stimulation was repeated at least 20 times (interval $\geq 10 \mathrm{~s}$ ) to assess variability of synaptic transmission at a given synapse. This time interval proved to be sufficiently long to avoid unintended short-term potentiation of synapses during the repetitions (Supplementary Fig. 5). A single 
train $(25 \mathrm{~Hz})$ of 24 bouton stimuli, each combined with a delayed (+ $10 \mathrm{~ms})$ bAP elicited in the postsynaptic cell, was applied to induce synaptic plasticity (Fig. 4b). This stimulation pattern resembles activity observed in granule cells in vivo ${ }^{40,70}$ and is known to involve postsynaptic mechanisms such as spike-timing-dependent plasticity ${ }^{38}$ at MF synapses ${ }^{46,47,53} .11$ out of 26 synapses were successfully re-evaluated 30 min after the induction protocol applying again at least 20 paired-pulse stimuli. Some synapses were additionally tested $15 \mathrm{~min}$ after the induction (Fig. 4d, e). One synapse was analysed per slice culture with the exception of those experiments aimed at analysing different MF synapses contacting the same mossy cell.

\section{Imaging and glutamate uncaging}

The recording chamber was integrated into a custom-made two-photon laser scanning microscope based on a BX50WI microscope frame and a Fluoview 300 scanning unit (Olympus, Hamburg, Germany). Excitation laser light was delivered through a $60 \mathrm{x}$ water immersion objective lens (NA 1.1, LUMF, Olympus). The excitation laser beams were expanded to overfill the back pupil of the objective at least twofold to achieve maximal spatial resolution. Fluorescence was detected by photomultiplier tubes (PMT, R3896; Hamamatsu Photonics, Herrsching, Germany) in non-descanned mode through the objective lens (green light: dichroic 680 DCXXR, 2 mm AR coated BG39; Schott, Mainz, Germany, and band-pass HQ 510/80, AHF analysentechnik, Tübingen, Germany) and an oil immersion condenser (NA 1.4, BX-UCDB-2, Olympus; green and red light: IR blocker HC-2P Emitter 680/SP, dichroic 560 DCXR, band pass HQ 510/80 and HQ 610/75, all from AHF analysentechnik). The amplified PMT output signals were digitized at $2.5 \mathrm{MHz}$ using a PC Board (NI PCl-6132, National Instruments). Scanning was controlled using Fluoview software (Olympus). Timing and data acquisition were accomplished using a 
program implemented in LabVIEW ${ }^{\mathrm{TM}}$ and the $\mathrm{NI}$ PCI-6251 board (National Instruments). The two-photon excitation source for imaging was a Ti:Sa laser tuned to $805 \mathrm{~nm}$ with a pulse repetition rate of $1 \mathrm{GHz}$ and a spectral full width at half maximum of $\sim 30 \mathrm{~nm}$ (Gigajet 20C; Laser Quantum, Konstanz, Germany; pump laser Verdi V5; Coherent, Dieburg, Germany; pulse length at focal plane $1.2 \mathrm{ps}$ ). Laser light for uncaging (720 nm, $76 \mathrm{MHz}$ pulse repetition rate, $~ 5 \mathrm{~nm}$ pulse width) was generated in a Mira 900F (Coherent; pump laser Verdi V10; pulse length at focal plane $0.4 \mathrm{ps}$ ). Excitation laser beams were combined using polarizing optics. A Keplerian telescope comprised of two biconvex lenses of $400 \mathrm{~mm}$ focal length was used to locate steering mirrors for both laser paths in front of the combining beam splitter in a conjugate plane to the scanning mirrors and the back focal plane of the objective lens, respectively ${ }^{71}$. This allowed convenient repositioning of the uncaging spot with respect to the imaging spot. These imaging conditions improved the signal to noise ratio due to a higher tolerable excitation level. We used point scans rather than line scans to gather maximal time resolution and to avoid dead times during recordings. As a result, individual recordings were analysed without averaging. Laser power was controlled by means of a half-lambda wave plate in the imaging laser path and an electro-optical modulator (Conoptics, via Polytec, Waldbronn, Germany) in the uncaging laser path. The dark current of the PMTs was measured over $100 \mathrm{~ms}$ before opening of the shutter (VS-25; Vincent Associates, via Acal BFi, Gröbenzell, Germany). Baseline fluorescence was collected for $200 \mathrm{~ms}$ before electrical and $300 \mathrm{~ms}$ before optical $(0.5 \mathrm{~ms})$ stimulation. Fluorescence response to the stimuli was monitored for $1 \mathrm{~s}$ (paired-pulse bouton stimulation), $1.5 \mathrm{~s}$ (uncaging), and $2.2 \mathrm{~s}$ (combined stimulation protocol). The uncaging laser spot was moved over the circumference of a spine to find the synaptic contact (no response if no hit). Background fluorescence was measured in the unlabelled tissue close to the labelled spines before stimulation onset. 
The dendritic distance was determined as dendritic path length between a synapse and the MC soma using z-projections of two-photon image stacks (voxel size: 0.184 × 0.184 × $1.0 \mu \mathrm{m}$; Supplementary Fig. 4a, b).

\section{Exclusion criteria for quantitative analysis}

Traces were excluded from analysis if one of the stated criteria was not met: Spontaneous AP or spontaneous synaptic event at the recorded synapse not closer than $0.1 \mathrm{~s}$ before the first stimulus, to allow for the determination of basal $\mathrm{Ca}^{2+}$-related fluorescence; maximal tolerated baseline fluorescence (green by red): 1.0; maximal tolerated postsynaptic holding current: $-0.5 \mathrm{nA}$; maximal tolerated postsynaptic membrane potential before first stimulus: $-60.0 \mathrm{mV}$.

\section{Analysis of EPSPs}

Voltage recordings were subjected to a smoothing procedure based on locally adaptive Bayesian P-splines (algorithm "FlexNEG") ${ }^{72}$ in order to reduce noise effectively without flattening of steep changes. Onset of EPSPs was determined iteratively using derivatives of decreasingly smoothened copies of nested intervals of a short time period ( $1-2 \mathrm{~ms}$ ) following the stimulus. The maximal slope of the rising EPSP (before AP firing in case of suprathreshold EPSPs) was determined similarly using decreasingly smoothened copies of nested intervals of the extracted rising phase. The threshold potential of synaptically evoked APs was defined as the inflection point of the rising phase of the AP, again obtained iteratively in nested intervals of a short time interval before AP peak. 


\section{Quantification of fluorescence intensity recordings}

Relative changes in fluorescence in response to stimulation were calculated as green fluorescence minus green baseline fluorescence divided by red fluorescence $(\Delta G / R)$, which is approximately proportional to the elevation of cytoplasmic $\left[\mathrm{Ca}^{2+}\right]$ over baseline ${ }^{63}$. Individual traces were smoothened by a $5 \mathrm{~ms}$ wide rectangular moving average filter. Traces were fitted to a sum of exponential peak functions ${ }^{73}$ using a genetic algorithm. Peak $\mathrm{Ca}^{2+}$ responses were determined separately for each stimulus as amplitudes of these peak functions.

\section{Statistics}

The Theil-Sen estimator of the regression slope and accordingly of the regression intercept was used for robust regression of EPSP slopes on peak $\mathrm{Ca}^{2+}$ responses (number of samples for regression estimate $=10000)$. A confidence interval $(0.025-0.975$, equivalent to $\alpha=0.05$ ) for Kendall's tau was obtained using the percentile bootstrap method (number of bootstrap samples $=1000$ ) as a robust estimate of correlation of parameters between synapses as well as within synapses, respectively ${ }^{74}$. Changes of fractions within categories of synaptic responses on paired-pulse stimulation following potentiation were tested under the null-hypothesis that there is no change using Wilcoxon signed-rank test with Pratts modification for zero differences $(\alpha=0.05$; function "wilcoxsign_test $(x \sim y$, distribution = "exact" , zero.method = c("Pratt")" from R-package "coin" ${ }^{\text {"5 }}$, called via R-script ${ }^{76}$ ). All procedures were implemented in LabVIEW ${ }^{\mathrm{TM}}$ (National Instruments). 


\section{Data availability}

The data that support the findings of this study are available from the corresponding author upon request.

\section{Acknowledgements}

We thank Prof. Peter Jonas, Prof. Thomas Oertner and Prof. Roger Nicoll for critically reading an earlier version of the manuscript, Prof. Imre Vida for helpful discussion, and Bettina Herde, Janice Graw and Dung Ludwig for excellent lab assistance.

Supported by the Deutsche Forschungsgemeinschaft (FOR 2419: FR 620/14-1) and Landesforschungsförderung Hamburg (LFF-FV27b) to M.F.; M.F. was Research Professor for Neuroscience of the Hertie Foundation.

\section{Author contributions}

A.D. designed the experiments, developed targeted "shadow" patching of MF boutons together with U.M., and performed all analyses and programming. U.M. performed the experiments combining bouton stimulation, mossy cell recording and two-photon $\mathrm{Ca}^{2+}$ imaging and contributed to experiment planning. A.T. performed the glutamate uncaging experiments. M.F. together with A.D. developed the concept of the paper and wrote the manuscript.

\section{Competing financial interests}

The authors declare no conflict of interest. 


\section{References}

1. Deguchi, Y., Donato, F., Galimberti, I., Cabuy, E. \& Caroni, P. Temporally matched subpopulations of selectively interconnected principal neurons in the hippocampus. Nat. Neurosci. 14, 495-504 (2011).

2. Blackstad, T.W. \& Kjaerheim, A. Special axo-dendritic synapses in the hippocampal cortex. Electron and light microscopic studies on the layer of mossy fibers. J. Comp. Neurol. 117, 133-159 (1961).

3. Hamlyn, L.H. The fine structure of the mossy fibre endings in the hippocampus of the rabbit. J. Anat. 96, 112-120 (1962).

4. Chicurel, M.E. \& Harris, K.M. Three-dimensional analysis of the structure and composition of CA3 branched dendritic spines and their synaptic relationships with mossy fiber boutons in the rat hippocampus. J. Comp. Neurol. 325, 169-182 (1992).

5. Rollenhagen, A. et al. Structural determinants of transmission at large hippocampal mossy fiber synapses. J. Neurosci. 27, 10434-10444 (2007).

6. Frotscher, M. Mossy fibres form synapses with identified pyramidal basket cells in the CA3 region of the guinea-pig hippocampus. A combined Golgi-electron microscope study. J. Neurocytol. 14, 245-259 (1985).

7. Acsady, L., Kamondi, A., Sik, A., Freund, T. \& Buzsaki, G. GABAergic cells are the major postsynaptic targets of mossy fibers in the rat hippocampus. J. Neurosci. 18, 3386-3403 (1998).

8. Scharfman, H.E. The enigmatic mossy cell of the dentate gyrus. Nat. Rev. Neurosci. 17, 562-575 (2016).

9. Frotscher, M., Seress, L., Schwerdtfeger, W.K. \& Buhl, E. The mossy cells of the fascia dentata: a comparative study of their fine structure and synaptic connections in rodents and primates. J. Comp. Neurol. 312, 145-163 (1991).

10. Scharfman, H.E., Kunkel, D.D. \& Schwartzkroin, P.A. Synaptic connections of dentate granule cells and hilar neurons: results of paired intracellular recordings and intracellular horseradish peroxidase injections. Neuroscience 37, 693-707 (1990).

11. Lysetskiy, M., Foldy, C. \& Soltesz, I. Long- and short-term plasticity at mossy fiber synapses on mossy cells in the rat dentate gyrus. Hippocampus 15, 691-696 (2005).

12. Patton, P.E. \& McNaughton, B. Connection matrix of the hippocampal formation: I. The dentate gyrus. Hippocampus 5, 245-286 (1995).

13. Duffy, A.M., Schaner, M.J., Chin, J. \& Scharfman, H.E. Expression of c-fos in hilar mossy cells of the dentate gyrus in vivo. Hippocampus 23, 649-655 (2013).

14. GoodSmith, D. et al. Spatial Representations of Granule Cells and Mossy Cells of the Dentate Gyrus. Neuron 93, 677-690.e5 (2017).

15. Danielson, N.B. et al. In Vivo Imaging of Dentate Gyrus Mossy Cells in Behaving Mice. Neuron 93, 552-559.e4 (2017).

16. Senzai, Y. \& Buzsaki, G. Physiological Properties and Behavioral Correlates of Hippocampal Granule Cells and Mossy Cells. Neuron 93, 691-704.e5 (2017). 
17. Soriano, E. \& Frotscher, M. Mossy cells of the rat fascia dentata are glutamateimmunoreactive. Hippocampus 4, 65-69 (1994).

18. Henze, D.A. \& Buzsaki, G. Hilar mossy cells: functional identification and activity in vivo. Prog. Brain Res. 163, 199-216 (2007).

19. Amaral, D.G. \& Lavenex, P. in The hippocampus book, edited by P. Andersen, R. Morris, D. Amaral, T. Bliss \& J. O'Keefe (Oxford University Press, New York, 2007), pp. 37-114.

20. Sloviter, R.S. \& Lomo, T. Updating the lamellar hypothesis of hippocampal organization. Front. Neural Circuits 6, 102 (2012).

21. Hashimotodani, Y. et al. LTP at Hilar Mossy Cell-Dentate Granule Cell Synapses Modulates Dentate Gyrus Output by Increasing Excitation/Inhibition Balance. Neuron 95, 928-943.e3 (2017).

22. Jinde, S. et al. Hilar mossy cell degeneration causes transient dentate granule cell hyperexcitability and impaired pattern separation. Neuron 76, 1189-1200 (2012).

23. Chancey, J.H., Poulsen, D.J., Wadiche, J.I. \& Overstreet-Wadiche, L. Hilar mossy cells provide the first glutamatergic synapses to adult-born dentate granule cells. $J$. Neurosci. 34, 2349-2354 (2014).

24. Galimberti, I. et al. Long-term rearrangements of hippocampal mossy fiber terminal connectivity in the adult regulated by experience. Neuron 50, 749-763 (2006).

25. Mori, M., Abegg, M.H., Gahwiler, B.H. \& Gerber, U. A frequency-dependent switch from inhibition to excitation in a hippocampal unitary circuit. Nature 431, 453-456 (2004).

26. Maruo, T., Mandai, K., Takai, Y. \& Mori, M. Activity-dependent alteration of the morphology of a hippocampal giant synapse. Mol. Cell. Neurosci. 71, 25-33 (2016).

27. Frotscher, M., Hamori, J. \& Wenzel, J. Transneuronal effects of entorhinal lesions in the early postnatal period on synaptogenesis in the hippocampus of the rat. Exp. Brain Res. 30, 549-560 (1977).

28. Bliss, T.V.P. \& Lomo, T. Long-lasting potentiation of synaptic transmission in the dentate area of the anaesthetized rabbit following stimulation of the perforant path. $J$. Physiol. 232, 331-356 (1973).

29. Bliss, T.V.P. \& Collingridge, G.L. A synaptic model of memory: long-term potentiation in the hippocampus. Nature 361, 31-39 (1993).

30. Whitlock, J.R., Heynen, A.J., Shuler, M.G. \& Bear, M.F. Learning induces long-term potentiation in the hippocampus. Science 313, 1093-1097 (2006).

31. Kirov, S.A., Sorra, K.E. \& Harris, K.M. Slices Have More Synapses than PerfusionFixed Hippocampus from both Young and Mature Rats. J. Neurosci. 19, 2876-2886 (1999).

32. Zhao, S., Forster, E., Chai, X. \& Frotscher, M. Different signals control laminar specificity of commissural and entorhinal fibers to the dentate gyrus. J. Neurosci. 23, $7351-7357$ (2003).

33. Frotscher, M. \& Gähwiler, B.H. Synaptic organization of intracellularly stained CA3 pyramidal neurons in slice cultures of rat hippocampus. Neuroscience 24, 541-551 (1988). 
34. Zhao, S. et al. Structural plasticity of hippocampal mossy fiber synapses as revealed by high-pressure freezing. J. Comp. Neurol. 520, 2340-2351 (2012).

35. Kitamura, K., Judkewitz, B., Kano, M., Denk, W. \& Hausser, M. Targeted patch-clamp recordings and single-cell electroporation of unlabeled neurons in vivo. Nat. Methods 5, 61-67 (2008).

36. Nicoll, R.A. \& Schmitz, D. Synaptic plasticity at hippocampal mossy fibre synapses. Nat. Rev. Neurosci. 6, 863-876 (2005).

37. Salin, P.A., Scanziani, M., Malenka, R.C. \& Nicoll, R.A. Distinct short-term plasticity at two excitatory synapses in the hippocampus. PNAS 93, 13304-13309 (1996).

38. Markram, H., Lubke, J., Frotscher, M. \& Sakmann, B. Regulation of synaptic efficacy by coincidence of postsynaptic APs and EPSPs. Science 275, 213-215 (1997).

39. McNaughton, B.L. \& Morris, R.G.M. Hippocampal synaptic enhancement and information storage within a distributed memory system. Trends Neurosci. 10, 408415 (1987).

40. Henze, D.A., Wittner, L. \& Buzsaki, G. Single granule cells reliably discharge targets in the hippocampal CA3 network in vivo. Nat. Neurosci. 5, 790-795 (2002).

41. Vyleta, N.P., Borges-Merjane, C. \& Jonas, P. Plasticity-dependent, full detonation at hippocampal mossy fiber-CA3 pyramidal neuron synapses. Elife 5, e17977 (2016).

42. Koester, H.J. \& Sakmann, B. Calcium dynamics in single spines during coincident preand postsynaptic activity depend on relative timing of back-propagating action potentials and subthreshold excitatory postsynaptic potentials. PNAS 95, 9596-9601 (1998).

43. Kovalchuk, Y., Eilers, J., Lisman, J. \& Konnerth, A. NMDA receptor-mediated subthreshold $\mathrm{Ca}(2+)$ signals in spines of hippocampal neurons. J. Neurosci. 20, 17911799 (2000).

44. Sabatini, B.L., Oertner, T.G. \& Svoboda, K. The life cycle of $\mathrm{Ca}(2+)$ ions in dendritic spines. Neuron 33, 439-452 (2002).

45. Yeckel, M.F., Kapur, A. \& Johnston, D. Multiple forms of LTP in hippocampal CA3 neurons use a common postsynaptic mechanism. Nat. Neurosci. 2, 625-633 (1999).

46. Kwon, H.-B. \& Castillo, P.E. Long-term potentiation selectively expressed by NMDA receptors at hippocampal mossy fiber synapses. Neuron 57, 108-120 (2008).

47. Rebola, N., Carta, M., Lanore, F., Blanchet, C. \& Mulle, C. NMDA receptor-dependent metaplasticity at hippocampal mossy fiber synapses. Nat. Neurosci. 14, 691-693 (2011).

48. Chamberland, S., Evstratova, A. \& Toth, K. Interplay between synchronization of multivesicular release and recruitment of additional release sites support short-term facilitation at hippocampal mossy fiber to CA3 pyramidal cells synapses. J. Neurosci. 34, 11032-11047 (2014).

49. Huang, C.-C., Chen, Y.-L., Liang, Y.-C. \& Hsu, K.-S. Role for cAMP and protein phosphatase in the presynaptic expression of mouse hippocampal mossy fibre depotentiation. J. Physiol. 543, 767-778 (2002). 
50. Ruiz, A., Campanac, E., Scott, R.S., Rusakov, D.A. \& Kullmann, D.M. Presynaptic GABAA receptors enhance transmission and LTP induction at hippocampal mossy fiber synapses. Nat. Neurosci. 13, 431-438 (2010).

51. Cheng, Q. \& Yakel, J.L. Presynaptic alpha7 nicotinic acetylcholine receptors enhance hippocampal mossy fiber glutamatergic transmission via PKA activation. J. Neurosci. 34, 124-133 (2014).

52. Rebola, N., Lujan, R., Cunha, R.A. \& Mulle, C. Adenosine A2A receptors are essential for long-term potentiation of NMDA-EPSCs at hippocampal mossy fiber synapses. Neuron 57, 121-134 (2008).

53. Astori, S., Pawlak, V. \& Kohr, G. Spike-timing-dependent plasticity in hippocampal CA3 neurons. J. Physiol. 588, 4475-4488 (2010).

54. Vergnano, A.M. et al. Zinc dynamics and action at excitatory synapses. Neuron $\mathbf{8 2}$, 1101-1114 (2014).

55. Montgomery, J.M. \& Madison, D.V. Discrete synaptic states define a major mechanism of synapse plasticity. Trends Neurosci. 27, 744-750 (2004).

56. Alle, H. \& Geiger, J.R.P. Combined analog and action potential coding in hippocampal mossy fibers. Science 311, 1290-1293 (2006).

57. Nakashiba, T. et al. Young dentate granule cells mediate pattern separation, whereas old granule cells facilitate pattern completion. Cell 149, 188-201 (2012).

58. Nakazawa, K. et al. Requirement for hippocampal CA3 NMDA receptors in associative memory recall. Science 297, 211-218 (2002).

59. Guzman, S.J., Schlogl, A., Frotscher, M. \& Jonas, P. Synaptic mechanisms of pattern completion in the hippocampal CA3 network. Science 353, 1117-1123 (2016).

60. Stoppini, L., Buchs, P.-A. \& Muller, D. A simple method for organotypic cultures of nervous tissue. J. Neurosci. Methods 37, 173-182 (1991).

61. Jonas, P., Major, G. \& Sakmann, B. Quantal components of unitary EPSCs at the mossy fibre synapse on CA3 pyramidal cells of rat hippocampus. J. Physiol. 472, 615663 (1993).

62. Maroteaux, M. \& Liu, S.J. Alteration of AMPA Receptor-Mediated Synaptic Transmission by Alexa Fluor 488 and 594 in Cerebellar Stellate Cells. eNeuro 3 (2016).

63. Yasuda, R. et al. Imaging calcium concentration dynamics in small neuronal compartments. Sci. STKE 2004, pl5 (2004).

64. Dodt, H.-U., Frick, A., Kampe, K. \& Zieglgansberger, W. NMDA and AMPA receptors on neocortical neurons are differentially distributed. Eur. J. Neurosci. 10, 3351-3357 (1998).

65. Riedemann, T., Polder, H.R. \& Sutor, B. Determination and compensation of series resistances during whole-cell patch-clamp recordings using an active bridge circuit and the phase-sensitive technique. Pflugers Arch. 468, 1725-1740 (2016).

66. Bischofberger, J., Engel, D., Li, L., Geiger, J.R.P. \& Jonas, P. Patch-clamp recording from mossy fiber terminals in hippocampal slices. Nat. Protoc. 1, 2075-2081 (2006).

67. Vyleta, N.P. \& Jonas, P. Loose coupling between Ca2+ channels and release sensors at a plastic hippocampal synapse. Science 343, 665-670 (2014). 
68. Claiborne, B.J., Amaral, D.G. \& Cowan, W.M. A light and electron microscopic analysis of the mossy fibers of the rat dentate gyrus. J. Comp. Neurol. 246, 435-458 (1986).

69. Schmidt-Hieber, C., Jonas, P. \& Bischofberger, J. Action potential initiation and propagation in hippocampal mossy fibre axons. J. Physiol. 586, 1849-1857 (2008).

70. Pernia-Andrade, A.J. \& Jonas, P. Theta-gamma-modulated synaptic currents in hippocampal granule cells in vivo define a mechanism for network oscillations. Neuron 81, 140-152 (2014).

71. Harvey, C.D. \& Svoboda, K. Locally dynamic synaptic learning rules in pyramidal neuron dendrites. Nature 450, 1195-1200 (2007).

72. Scheipl, F. \& Kneib, T. Locally adaptive Bayesian P-splines with a Normal-ExponentialGamma prior. Comput. Stat. Data Anal. 53, 3533-3552 (2009).

73. Richardson, M.J.E. \& Silberberg, G. Measurement and analysis of postsynaptic potentials using a novel voltage-deconvolution method. J. Neurophysiol. 99, 10201031 (2008).

74. Wilcox, R.R. Introduction to robust estimation and hypothesis testing. 3rd ed. (Academic Press, San Diego, 2012).

75. Hothorn, T., Hornik, K., van de Wiel, M.A. \& Zeileis, A. Implementing a Class of Permutation Tests. The coin Package. J. Stat. Soft. 28 (2008).

76. $\mathrm{R}$ Development Core Team. $R$ : A language and environment for statistical computing (R Foundation for Statistical Computing, Vienna, Austria, 2017). 


\section{Legends to Tables and Figures}

Table 1 Classification of responses to paired-pulse stimulation.

Response patterns are shown schematically on top (category 1: both responses are subthreshold; category 2: only the response to the second stimulus is suprathreshold; category 3: only the response to the first stimulus is suprathreshold; category 4: both responses are suprathreshold). Each synapse is represented in all 4 response categories (fractions summing up to 1). Coding of synapses by coloured symbols as in Fig. 2. Bottom: Mean \pm s.e.m. of fractions of all 26 synapses.

Table 2 Change of response categories following potentiation.

Response patterns are shown schematically on top (categories as in Table 1). Each synapse is represented in all 4 response categories (changes of fractions summing up to zero). Coding of synapses by coloured symbols as in Fig. 2. Bottom: Mean \pm s.e.m. of fraction changes of the 11 synapses analysed.

$\left.{ }^{*}\right)$ significant increase in category 4 after potentiation (Wilcoxon signed-rank test with Pratts modification, $\mathrm{n}=11, \mathrm{P}=0.03125)$.

Fig. 1 Interrogation of single MF-MC synapses. a MC filled with Alexa 594 dextran and the $\mathrm{Ca}^{2+}$-sensitive dye Fluo-4FF via a patch pipette (arrow). Scale bar: $20 \mu \mathrm{m}$. Boxed area shown in d. b Schematic diagram illustrating stimulation and recording sites. Arrow: MF bouton; yellow cross: imaging site of $\mathrm{Ca}^{2+}$ transients; green: extracellular tracer (Alexa 488 hydrazide). c Two-photon XY-frame to illustrate the bouton "shadow" (arrow) before patching. Scale bar: $5 \mu \mathrm{m}$. d Boxed area in a. Boutons presynaptic to the two encircled spines were stimulated and voltage responses recorded at the somatic pipette. Simultaneously, $\mathrm{Ca}^{2+}$ transients were recorded in the spines. Scale bar: $5 \mu \mathrm{m}$. e, f Unitary 
voltage responses and $\mathrm{Ca}^{2+}$ transients evoked by paired-pulse stimulation (orange dotted lines) of MF synapses on spine 1 and 2, respectively. Blue and green lines: Medians of all repetitions; grey lines: Individual trials.

Fig. 2 Functional heterogeneity of MF-MC synapses. a Unitary voltage and $\mathrm{Ca}^{2+}$ responses to the first of the paired stimuli in three MF-MC synapses from three different slice cultures (synapse 3 , synapse 10, and synapse 23). (•) subthreshold responses, ( $\mathbf{\Delta})$ suprathreshold responses. Lines represent regression of peak $\mathrm{Ca}^{2+}$ amplitudes on EPSP slopes in the three synapses (separate regression lines for suband suprathreshold responses for synapse 10, showing supralinear $\mathrm{Ca}^{2+}$ rises if postsynaptic APs were generated). Sub: subthreshold response; supra: suprathreshold response. b Medians of all responses obtained in each of the 26 synapses from 26 different slice cultures, bars representing median absolute deviations from the median.

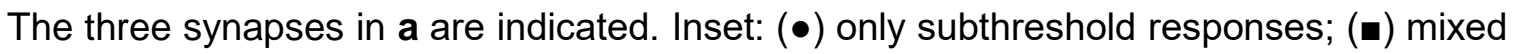
responses; ( $\Delta$ ) only suprathreshold responses to the first stimulus. Blue line represents regression of peak $\mathrm{Ca}^{2+}$ amplitude on EPSP slope, right panel shows the correlation between these parameters. The bootstrap mean $(\downarrow)$ of Kendalls tau and a confidence interval $(0.025-0.975$, vertical bars) is shown as a robust measure of correlation. If 0 (red line) is not included in the confidence interval, the parameters are considered to be correlated $\left({ }^{*}\right)$ at a significance level of 0.05 . c Paired-pulse ratios (PPR) of EPSP slope versus EPSP slope of the 26 synapses (medians of all repetitions per synapse). Red line indicates a PPR of 1. Blue line represents regression of PPR of EPSP slope on EPSP slope. PPF and PPD: Paired-pulse facilitation and depression. Right panel shows the correlation between PPR of EPSP slope and EPSP slope of these synapses. 
Fig. 3 Heterogeneity of postsynaptic responses to glutamate uncaging. a Hilar mossy cell filled with Alexa 594 (red) and Fluo-5F for $\mathrm{Ca}^{2+}$ imaging (arrow points to patch pipette). Enlarged boxed area shows positions of imaging (yellow cross) and uncaging (orange cross) laser spots in the postsynaptic spine and the synaptic cleft, respectively. Glutamate was photo-released at the synaptic cleft from MNI-glutamate using two-photon uncaging. Scale bars: $20 \mu \mathrm{m}$ and $5 \mu \mathrm{m}$, respectively. b, c Each synapse was probed with six laser pulses of increasing intensities, resulting in increasing amplitudes of uncPSPs and $\mathrm{Ca}^{2+}$ transients in the spine (responses to three laser pulses are shown). $\mathbf{d}$ Voltage and $\mathrm{Ca}^{2+}$ responses to increasing intensities (dots). Lines represent regression of peak $\mathrm{Ca}^{2+}$ amplitudes on uncPSP amplitudes in selected synapses (different colours). e Regression lines of all 79 synapses studied (in 79 slice cultures). f Application of the AMPA receptor antagonist CNQX diminished voltage responses $(n=8)$. g Application of the NMDA receptor antagonist D-APV drastically reduced the amplitudes of Ca ${ }^{2+}$ transients $(n=23)$.

Fig. 4 Potentiation of individual identified MF-MC synapses. a Unitary voltage (top) and $\mathrm{Ca}^{2+}$ response (bottom) to paired-pulse stimulation of a synapse prior to potentiation (synapse no. 3; blue and green lines: Medians of all repetitions). b Induction of plasticity in the synapse in a by combining bouton stimulation and backpropagating action potentials (bAPs); inset: first three responses. Orange dotted lines: Bouton stimulations; magenta lines: Postsynaptic stimuli (bAPs). Dramatic increase in $\mathrm{Ca}^{2+}$ during combined stimulation (green line) but not in response to a train of only bAPs (black line). c Same synapse as in a, 30 min after combined stimulation. $\mathbf{d}$ Time course relative to the induction train. EPSP slope in response to the first of paired-pulse stimuli, $(\bullet)$ subthreshold responses, (ム) suprathreshold responses; headlines: Numbers of sub- and suprathreshold 
responses, fraction of suprathreshold responses. e Time course of paired-pulse ratio of EPSP slope of the responses in $\mathbf{d}$; red lines indicate a paired-pulse ratio of 1.

Fig. 5 Heterogeneous synaptic plasticity of MF-MC synapses. a Percent change of slope of EPSP and peak $\mathrm{Ca}^{2+}$ amplitude in response to the first stimulus $30 \mathrm{~min}$ after potentiation in a subset of synapses; coding as in Fig. 2 (indicating the synaptic state prior to potentiation; medians of all repetitions per synapse). The synapses no. 3, 10, and 23 are marked as in Fig. 2. Blue line represents regression of percent change of peak $\mathrm{Ca}^{2+}$ on percent change of EPSP slope. Right panel shows the correlation between these parameters. The bootstrap mean $(\bullet)$ of Kendalls tau and a confidence interval $(0.025-0.975$, vertical bars) is shown as a robust measure of correlation. If 0 (red line) is not included in the confidence interval, the parameters are considered to be correlated $\left(^{*}\right)$ at a significance level of 0.05 . b Percent change of paired-pulse ratio of EPSP slope versus change of EPSP slope $30 \mathrm{~min}$ after potentiation of these synapses. Blue line represents regression, right panel shows the correlation between these parameters; PPF: Paired-pulse facilitation. c Percent change of EPSP slope 30 min after potentiation versus initial EPSP slope of these synapses. Blue line represents regression, right panel shows the correlation between these parameters. d Percent change of EPSP slope $30 \mathrm{~min}$ after potentiation versus initial peak $\mathrm{Ca}^{2+}$ response of these synapses. Blue line represents regression, right panel shows the correlation between these parameters. e Percent change of EPSP slope $30 \mathrm{~min}$ after potentiation versus initial fraction of suprathreshold EPSPs of these synapses. Blue line represents regression, right panel shows the correlation between these parameters. $f$ Change of response categories of these synapses. 
Fig. 6 MF-MC synapses represent a metastable network switch. a If the synapse (open arrow) is in subthreshold mode, only the MC (red) will notice AP firing of the presynaptic granule cell (black). b In suprathreshold, detonating mode, granule cells and interneurons (blue) postsynaptic to the MC will be notified of this unitary MF-MC EPSP. c MF-MC synapses are encountered in three different states with respect to MC detonation. Stable non-detonator synapses show exclusively subthreshold responses. Metastable detonator synapses are on the edge to detonation. They may become facilitated or depressed and are dependent on AP frequency and accompanying presynaptic EPSPs ${ }^{56}$. Stable full detonator synapses consistently elicit AP firing of the MC (as illustrated in $\mathbf{b}$ ). 


\begin{tabular}{|c|c|c|c|c|c|}
\hline Synapse & $\begin{array}{l}\text { No. } \\
\text { of } \\
\text { trials }\end{array}$ & $\begin{array}{l}\text { Fraction of } \\
\text { trials in } \\
\text { category } 1\end{array}$ & $\begin{array}{l}\text { Fraction of } \\
\text { trials in } \\
\text { category } 2\end{array}$ & $\begin{array}{l}\text { Fraction of } \\
\text { trials in } \\
\text { category } 3\end{array}$ & $\begin{array}{l}\text { Fraction of } \\
\text { trials in } \\
\text { category } 4\end{array}$ \\
\hline - 1 & 20 & 1 & 0 & 0 & 0 \\
\hline - 2 & 23 & 0.70 & 0.30 & 0 & 0 \\
\hline - 3 & 19 & 0.89 & 0.11 & 0 & 0 \\
\hline - 4 & 15 & 0.87 & 0.13 & 0 & 0 \\
\hline 5 & 22 & 0.24 & 0.71 & 0.05 & 0 \\
\hline-6 & 19 & 0.05 & 0.63 & 0 & 0.32 \\
\hline 7 & 19 & 0.42 & 0.21 & 0.11 & 0.26 \\
\hline$\square$ & 18 & 0 & 0.61 & 0 & 0.39 \\
\hline 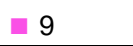 & 10 & 0.20 & 0.40 & 0 & 0.40 \\
\hline 10 & 37 & 0.26 & 0.29 & 0.03 & 0.43 \\
\hline 11 & 19 & 0.29 & 0.24 & 0.06 & 0.41 \\
\hline - 12 & 16 & 0 & 0.62 & 0 & 0.37 \\
\hline - 13 & 19 & 0 & 0.32 & 0 & 0.68 \\
\hline - 14 & 20 & 0.11 & 0.11 & 0.63 & 0.16 \\
\hline - 15 & 20 & 0 & 0.15 & 0 & 0.85 \\
\hline - 16 & 19 & 0 & 0.11 & 0 & 0.89 \\
\hline a 17 & 20 & 0 & 0.15 & 0 & 0.85 \\
\hline ㅁ 18 & 38 & 0 & 0.05 & 0 & 0.95 \\
\hline . 19 & 40 & 0 & 0.05 & 0 & 0.95 \\
\hline$\triangle 20$ & 16 & 0 & 0 & 0 & 1 \\
\hline$\triangle 21$ & 11 & 0 & 0 & 0 & 1 \\
\hline$\Delta 22$ & 20 & 0 & 0 & 0 & 1 \\
\hline$\Delta 23$ & 22 & 0 & 0 & 0 & 1 \\
\hline$\Delta 24$ & 20 & 0 & 0 & 0 & 1 \\
\hline$\Delta 25$ & 19 & 0 & 0 & 0 & 1 \\
\hline$\Delta 26$ & 38 & 0 & 0 & 0 & 1 \\
\hline Mean & - & 0.19 & 0.20 & 0.03 & 0.57 \\
\hline s.e.m. & - & 0.062 & 0.044 & 0.024 & 0.078 \\
\hline
\end{tabular}

Table 1 Classification of responses to paired-pulse stimulation.

Response patterns are shown schematically on top (category 1: both responses are subthreshold; category 2: only the response to the second stimulus is suprathreshold; category 3: only the response to the first stimulus is suprathreshold; category 4: both responses are suprathreshold). Each synapse is represented in all 4 response categories (fractions summing up to 1). Coding of synapses by coloured symbols as in Fig. 2. Bottom: Mean \pm s.e.m. of fractions of all 26 synapses. 


\begin{tabular}{|c|c|c|c|c|c|c|}
\hline Synapse & $\begin{array}{l}\text { No. of } \\
\text { trials } \\
\text { before } \\
\text { potent. }\end{array}$ & $\begin{array}{l}\text { No. of } \\
\text { trials } \\
\text { after } \\
\text { potent. }\end{array}$ & $\begin{array}{l}\text { Change of } \\
\text { fraction in } \\
\text { category } 1\end{array}$ & $\begin{array}{l}\text { Change of } \\
\text { fraction in } \\
\text { category } 2\end{array}$ & $\begin{array}{l}\text { Change of } \\
\text { fraction in } \\
\text { category } 3\end{array}$ & $\begin{array}{l}\text { Change of } \\
\text { fraction in } \\
\text { category } 4\end{array}$ \\
\hline 1 & 20 & 27 & 0 & 0 & 0 & 0 \\
\hline - 3 & 19 & 19 & -0.58 & 0.37 & 0.11 & 0.11 \\
\hline 10 & 37 & 18 & -0.26 & -0.29 & -0.03 & 0.57 \\
\hline 11 & 19 & 19 & -0.29 & -0.24 & -0.06 & 0.59 \\
\hline 13 & 19 & 33 & 0 & -0.22 & 0 & 0.22 \\
\hline 15 & 20 & 20 & 0 & -0.15 & 0 & 0.15 \\
\hline — 17 & 20 & 20 & 0 & -0.15 & 0 & 0.15 \\
\hline$\triangle 20$ & 16 & 15 & 0 & 0 & 0 & 0 \\
\hline$\Delta 23$ & 22 & 24 & 0 & 0 & 0 & 0 \\
\hline$\Delta 24$ & 20 & 19 & 0 & 0 & 0 & 0 \\
\hline$\Delta 25$ & 19 & 20 & 0 & 0.05 & 0 & -0.05 \\
\hline Mean & - & - & -0.10 & -0.06 & 0.002 & $0.16^{*}$ \\
\hline s.e.m. & - & - & 0.058 & 0.055 & 0.012 & 0.068 \\
\hline
\end{tabular}

Table 2 Change of response categories following potentiation.

Response patterns are shown schematically on top (categories as in Table 1). Each synapse is represented in all 4 response categories (changes of fractions summing up to zero). Coding of synapses by coloured symbols as in Fig. 2. Bottom: Mean \pm s.e.m. of fraction changes of the 11 synapses analysed.

${ }^{*}$ ) significant increase in category 4 after potentiation (Wilcoxon signed-rank test with Pratts modification, $n=11, P=0.03125$ ). 
a

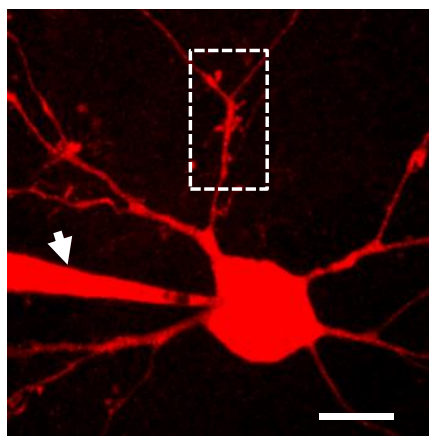

d

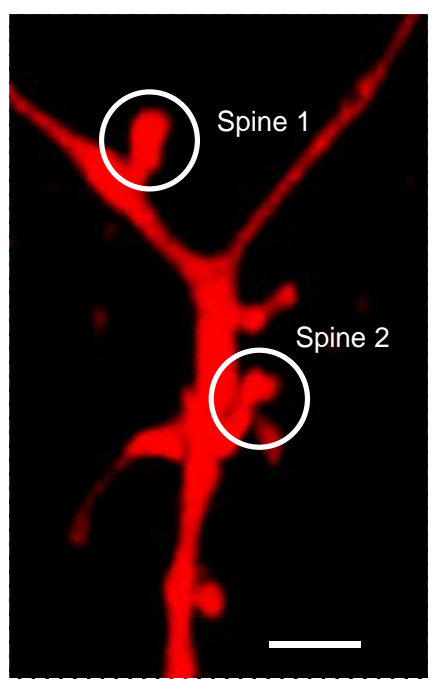

b

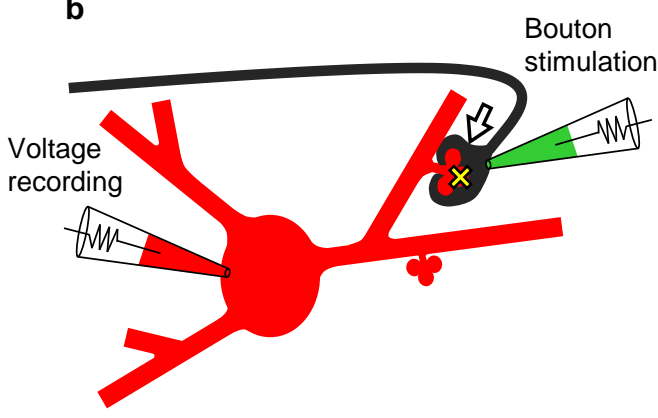

e

Spine 1

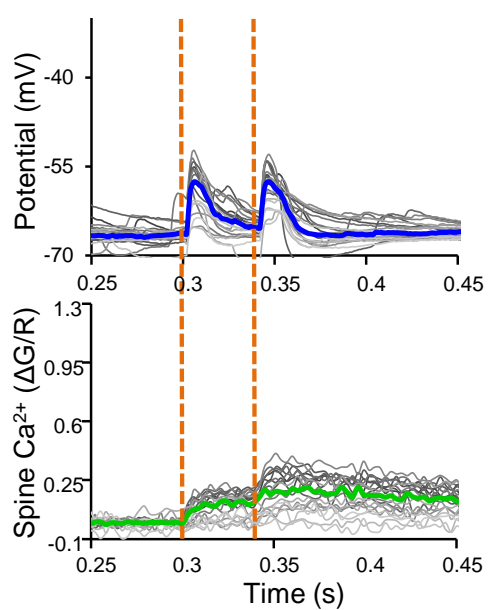

c

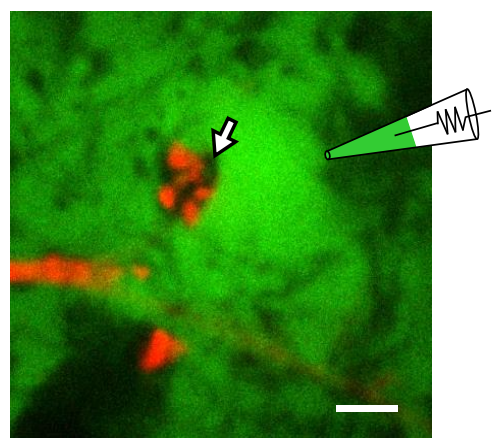

f

Spine 2

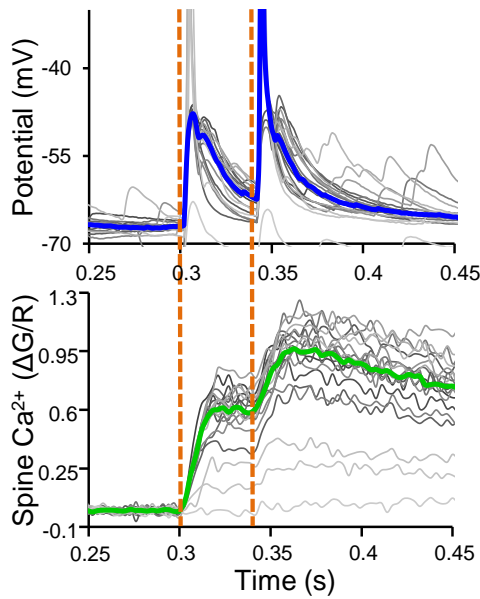

Fig. 1 Interrogation of single MF-MC synapses. a MC filled with Alexa 594 dextran and the $\mathrm{Ca}^{2+}$-sensitive dye Fluo-4FF via a patch pipette (arrow). Scale bar: $20 \mu \mathrm{m}$. Boxed area shown in d. b Schematic diagram illustrating stimulation and recording sites. Arrow: MF bouton; yellow cross: imaging site of $\mathrm{Ca}^{2+}$ transients; green: extracellular tracer (Alexa 488 hydrazide). c Two-photon XY-frame to illustrate the bouton "shadow" (arrow) before patching. Scale bar: $5 \mu \mathrm{m}$. d Boxed area in a. Boutons presynaptic to the two encircled spines were stimulated and voltage responses recorded at the somatic pipette. Simultaneously, $\mathrm{Ca}^{2+}$ transients were recorded in the spines. Scale bar: $5 \mu \mathrm{m}$. e, f Unitary voltage responses and $\mathrm{Ca}^{2+}$ transients evoked by paired-pulse stimulation (orange dotted lines) of MF synapses on spine 1 and 2, respectively. Blue and green lines: Medians of all repetitions; grey lines: Individual trials. 
a Individual responses to the first stimulus in three synapses
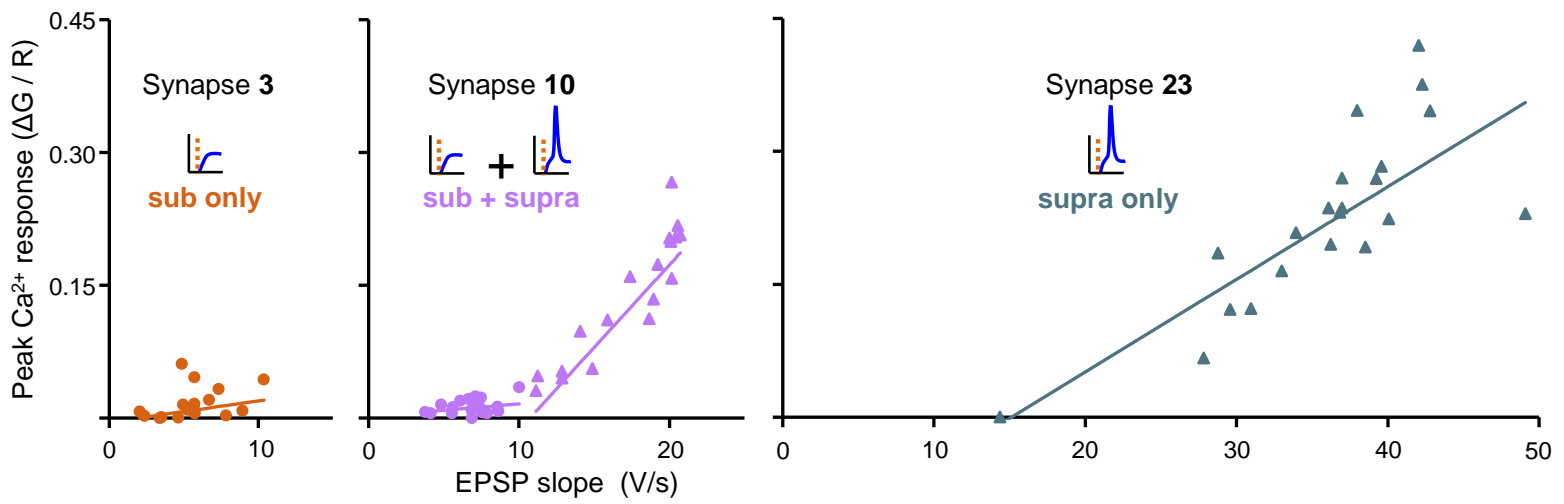

b Median responses to the first stimulus in 26 synapses

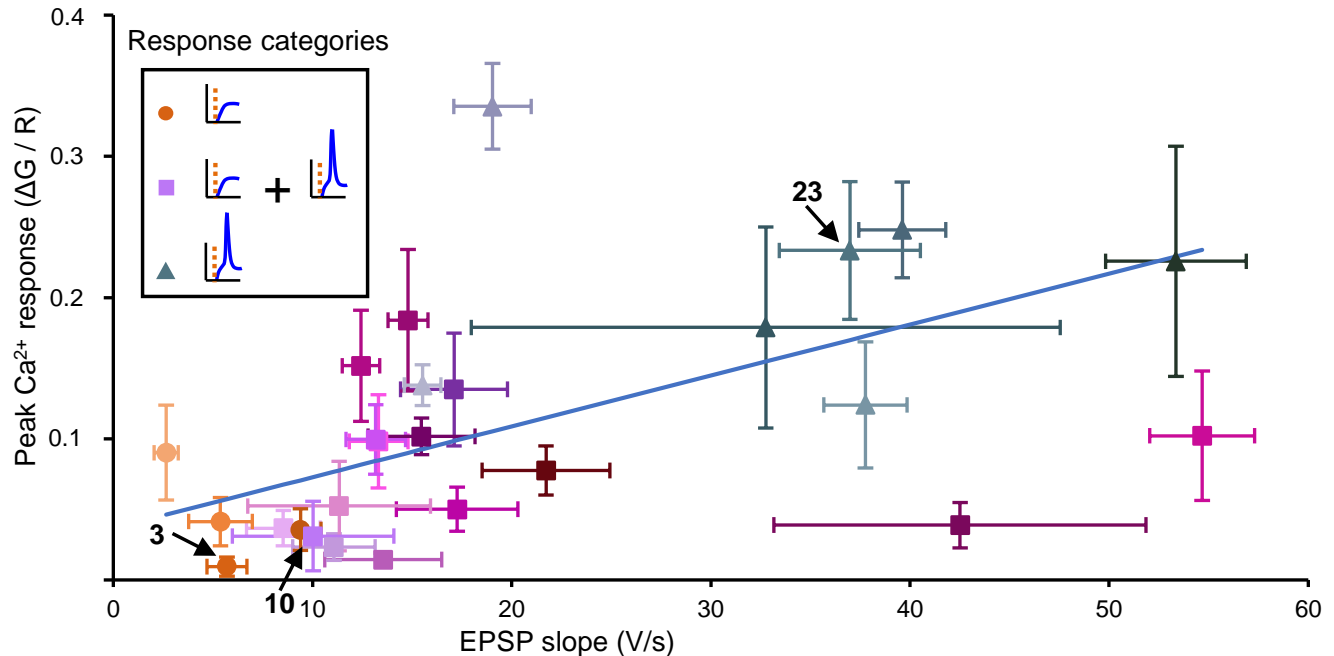

Correlation

Paired-pulse ratio (second by first response)

Correlation
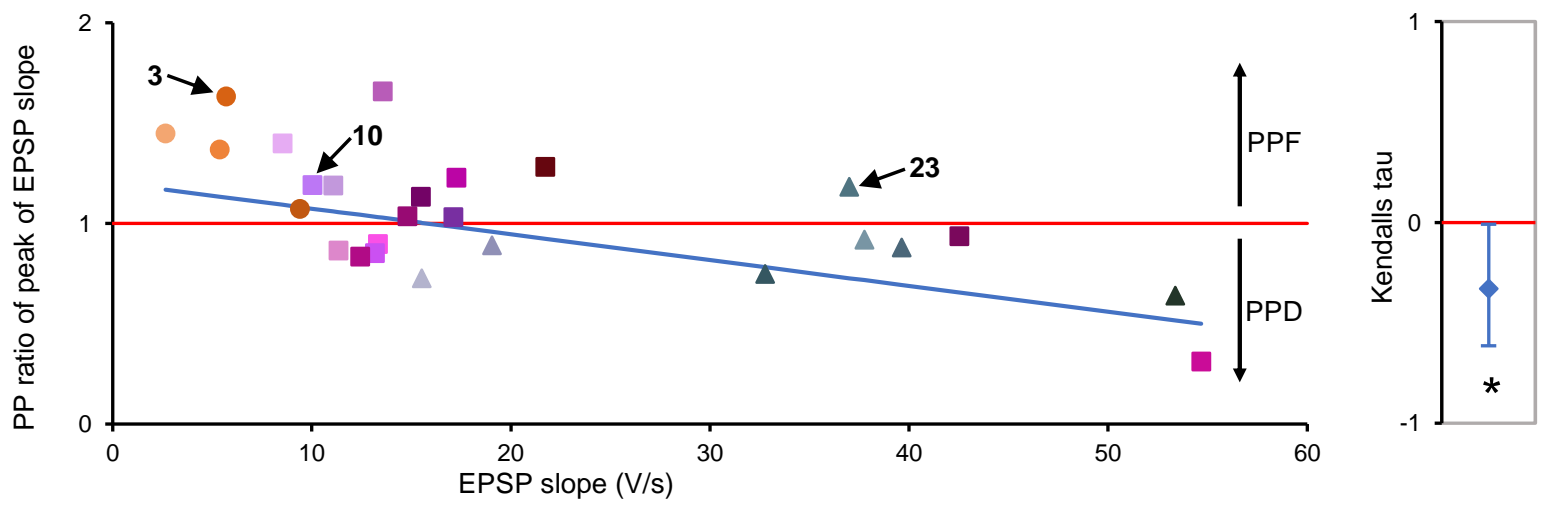
Fig. 2 Functional heterogeneity of MF-MC synapses. a Unitary voltage and $\mathrm{Ca}^{2+}$ responses to the first of the paired stimuli in three MF-MC synapses from three different slice cultures (synapse 3 , synapse 10, and synapse 23). (•) subthreshold responses, ( $(\mathbf{\Delta})$ suprathreshold responses. Lines represent regression of peak $\mathrm{Ca}^{2+}$ amplitudes on EPSP slopes in the three synapses (separate regression lines for suband suprathreshold responses for synapse 10, showing supralinear $\mathrm{Ca}^{2+}$ rises if postsynaptic APs were generated). Sub: subthreshold response; supra: suprathreshold response. b Medians of all responses obtained in each of the 26 synapses from 26 different slice cultures, bars representing median absolute deviations from the median. The three synapses in a are indicated. Inset: (•) only subthreshold responses; ( $\mathbf{a})$ mixed responses; ( $\mathbf{\Delta}$ ) only suprathreshold responses to the first stimulus. Blue line represents regression of peak $\mathrm{Ca}^{2+}$ amplitude on EPSP slope, right panel shows the correlation between these parameters. The bootstrap mean ( $\downarrow$ ) of Kendalls tau and a confidence interval $(0.025-0.975$, vertical bars) is shown as a robust measure of correlation. If 0 (red line) is not included in the confidence interval, the parameters are considered to be correlated $\left({ }^{*}\right)$ at a significance level of 0.05 . c Paired-pulse ratios (PPR) of EPSP slope versus EPSP slope of the 26 synapses (medians of all repetitions per synapse). Red line indicates a PPR of 1. Blue line represents regression of PPR of EPSP slope on EPSP slope. PPF and PPD: Paired-pulse facilitation and depression. Right panel shows the correlation between PPR of EPSP slope and EPSP slope of these synapses. 
a
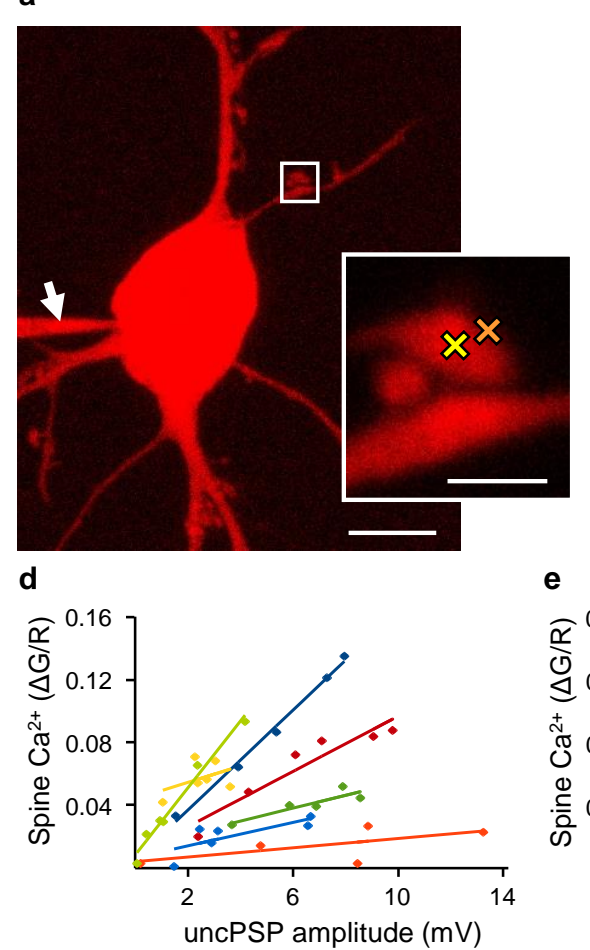

f

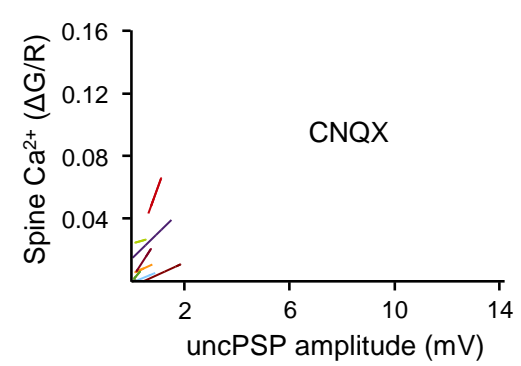

b
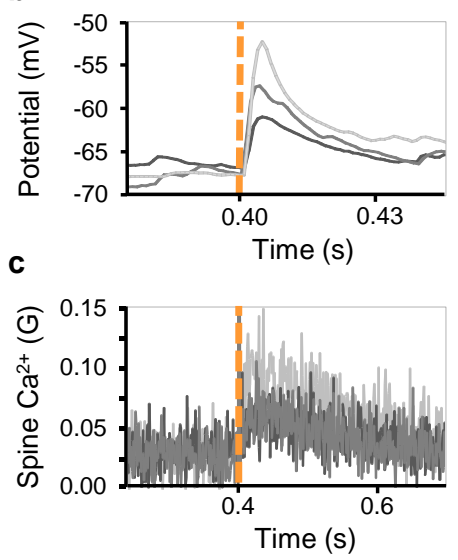

e

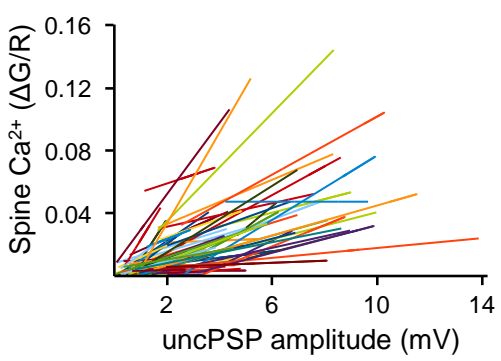

g

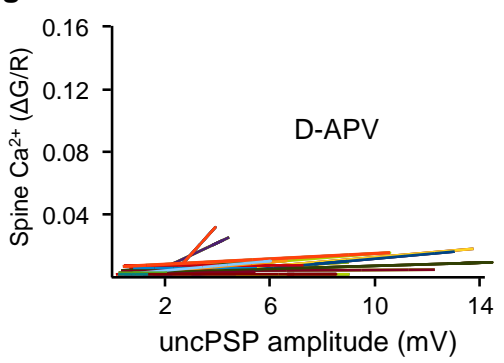

Fig. 3 Heterogeneity of postsynaptic responses to glutamate uncaging. a Hilar mossy cell filled with Alexa 594 (red) and Fluo-5F for $\mathrm{Ca}^{2+}$ imaging (arrow points to patch pipette). Enlarged boxed area shows positions of imaging (yellow cross) and uncaging (orange cross) laser spots in the postsynaptic spine and the synaptic cleft, respectively. Glutamate was photo-released at the synaptic cleft from MNI-glutamate using two-photon uncaging. Scale bars: $20 \mu \mathrm{m}$ and $5 \mu \mathrm{m}$, respectively. b, c Each synapse was probed with six laser pulses of increasing intensities, resulting in increasing amplitudes of uncPSPs and $\mathrm{Ca}^{2+}$ transients in the spine (responses to three laser pulses are shown). $\mathbf{d}$ Voltage and $\mathrm{Ca}^{2+}$ responses to increasing intensities (dots). Lines represent regression of peak $\mathrm{Ca}^{2+}$ amplitudes on uncPSP amplitudes in selected synapses (different colours). e Regression lines of all 79 synapses studied (in 79 slice cultures). f Application of the AMPA receptor antagonist CNQX diminished voltage responses $(n=8)$. $\mathbf{g}$ Application of the NMDA receptor antagonist D-APV drastically reduced the amplitudes of $\mathrm{Ca}^{2+}$ transients $(n=23)$. 
a Before potentiation

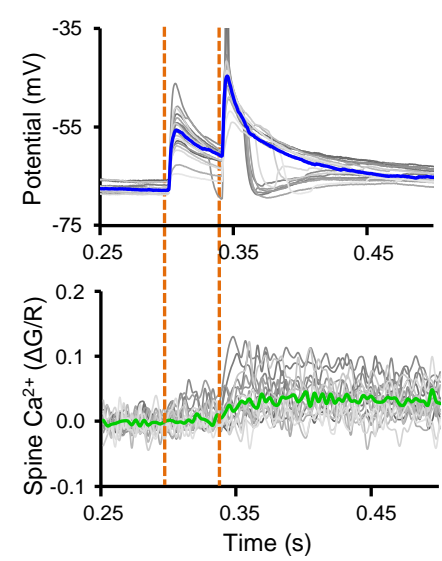

b
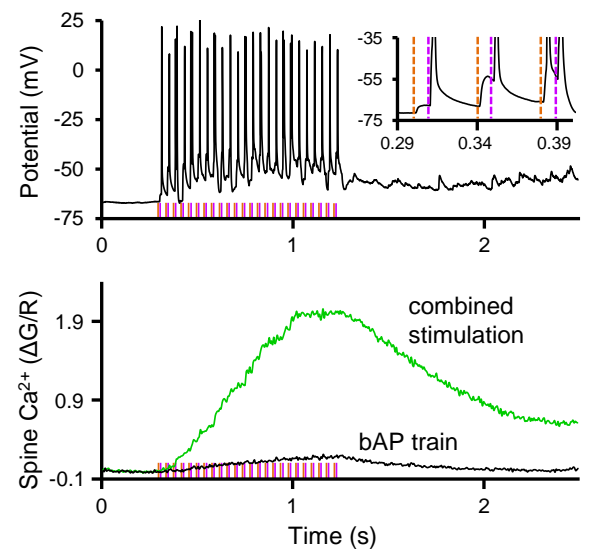

d Time course of EPSP slope (first response)

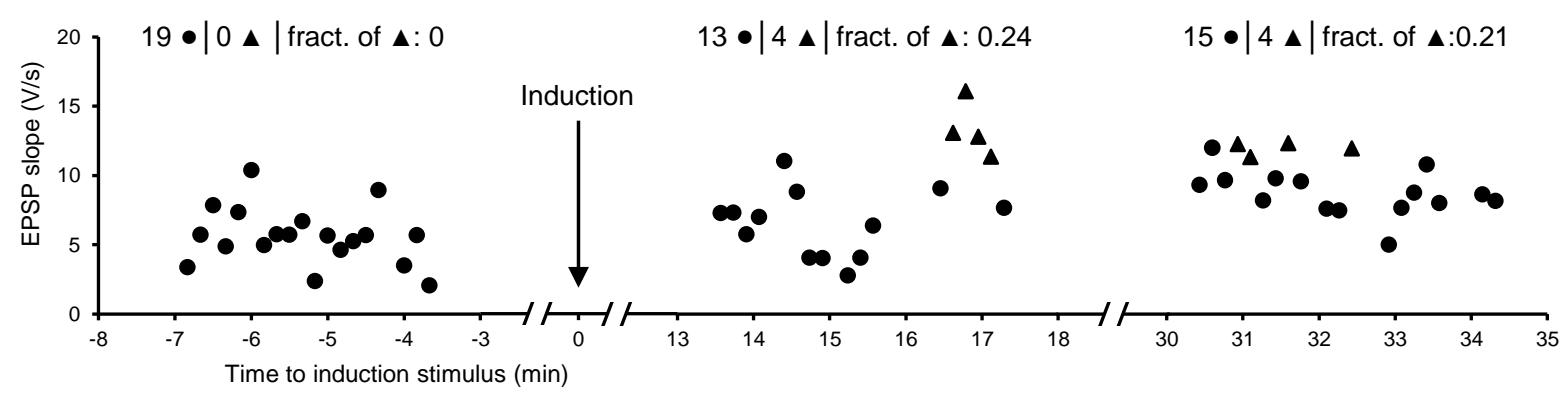

e Time course of paired-pulse ratio of EPSP slope

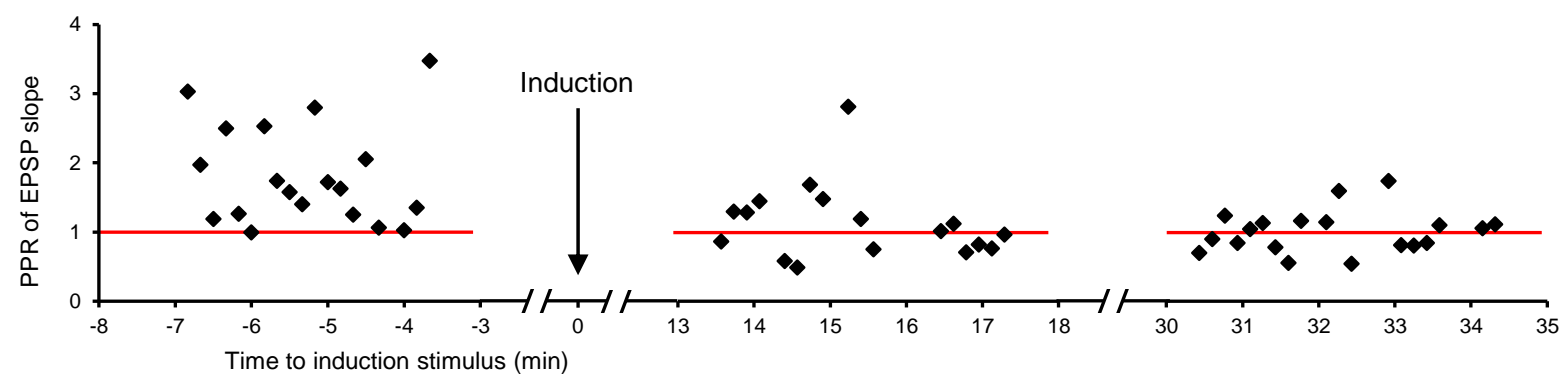

Fig. 4 Potentiation of individual identified MF-MC synapses. a Unitary voltage (top) and $\mathrm{Ca}^{2+}$ response (bottom) to paired-pulse stimulation of a synapse prior to potentiation (synapse no. 3; blue and green lines: Medians of all repetitions). b Induction of plasticity in the synapse in a by combining bouton stimulation and backpropagating action potentials (bAPs); inset: first three responses. Orange dotted lines: Bouton stimulations; magenta lines: Postsynaptic stimuli (bAPs). Dramatic increase in $\mathrm{Ca}^{2+}$ during combined stimulation (green line) but not in response to a train of only bAPs (black line). c Same synapse as in a, 30 min after combined stimulation. $\mathbf{d}$ Time course relative to the induction train. EPSP slope in response to the first of paired-pulse stimuli, $(\bullet)$ subthreshold responses, (A) suprathreshold responses; headlines: Numbers of sub- and suprathreshold responses, fraction of suprathreshold responses. e Time course of paired-pulse ratio of EPSP slope of the responses in $\mathbf{d}$; red lines indicate a paired-pulse ratio of 1 . 
a Change of response to first stimulus

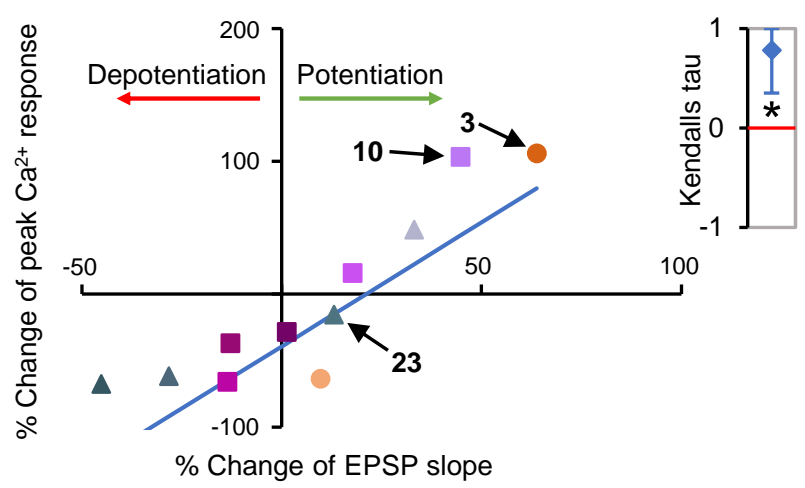

C Change of EPSP slope versus initial EPSP slope

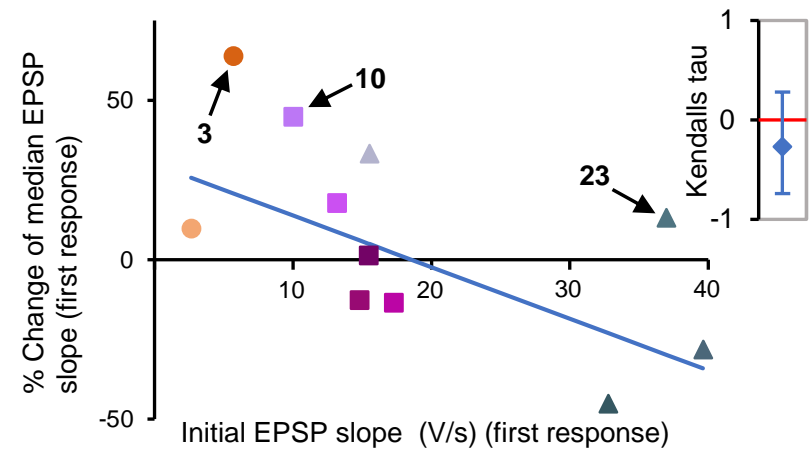

e

Change of EPSP slope versus initial fraction of suprathreshold EPSPs

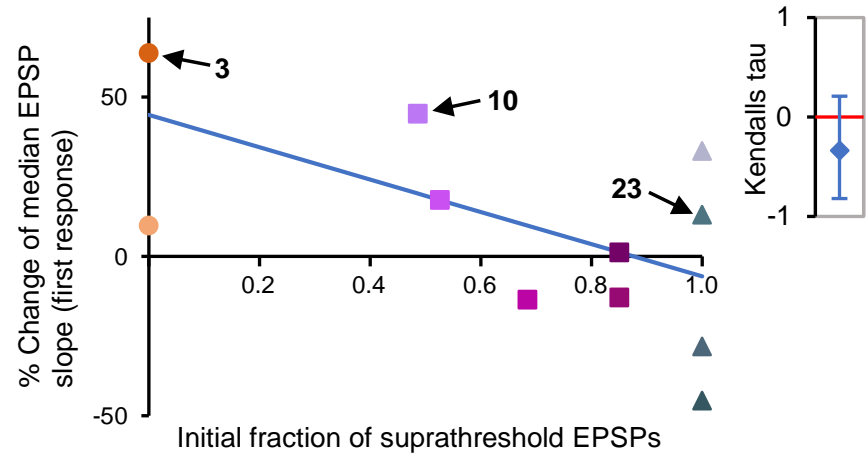

b Change of paired-pulse ratio versus change of EPSP slope

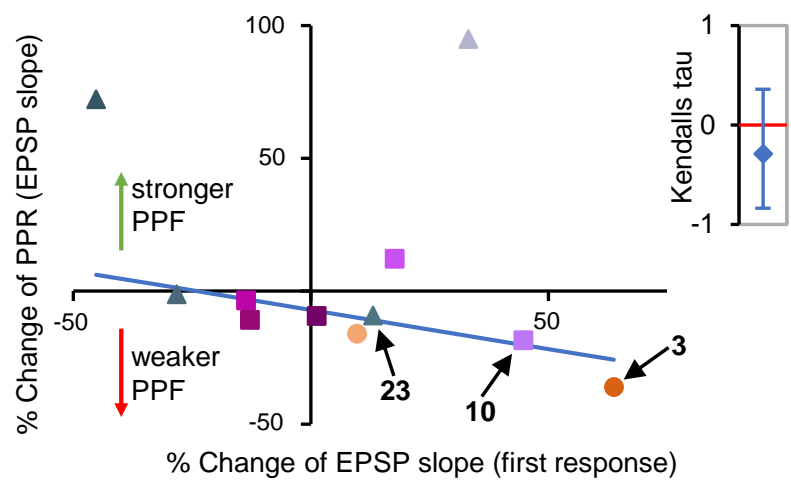

d Change of EPSP slope versus initial peak $\mathrm{Ca}^{2+}$ response

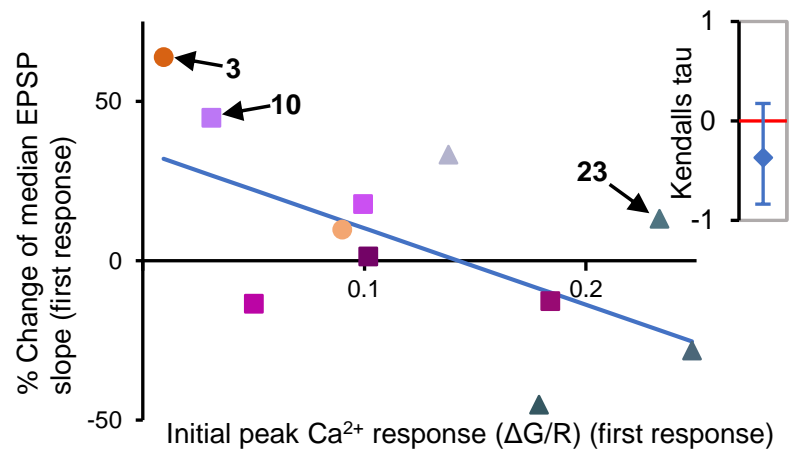

f

Change of response categories to first stimulus

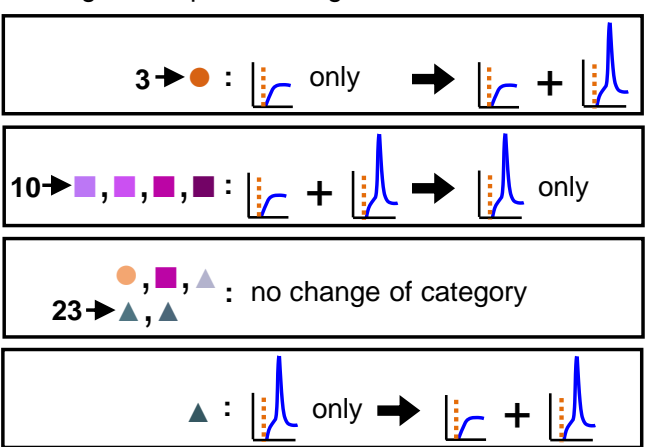

Fig. 5 Heterogeneous synaptic plasticity of MF-MC synapses. a Percent change of slope of EPSP and peak $\mathrm{Ca}^{2+}$ amplitude in response to the first stimulus 30 min after potentiation in a subset of synapses; coding as in Fig. 2 (indicating the synaptic state prior to potentiation; medians of all repetitions per synapse). The synapses no. 3, 10, and 23 are marked as in Fig. 2. Blue line represents regression of percent change of peak $\mathrm{Ca}^{2+}$ on percent change of EPSP slope. Right panel shows the correlation between these parameters. The bootstrap mean ( $\bullet$ ) of Kendalls tau and a confidence interval $(0.025-0.975$, vertical bars) is shown as a robust measure of correlation. If 0 (red line) is not included in the confidence interval, the parameters are considered to be correlated $\left(^{*}\right)$ at a significance level of 0.05 . b Percent change of paired-pulse ratio of EPSP slope versus change of EPSP slope $30 \mathrm{~min}$ after potentiation of these synapses. Blue line 
represents regression, right panel shows the correlation between these parameters; PPF: Paired-pulse facilitation. c Percent change of EPSP slope 30 min after potentiation versus initial EPSP slope of these synapses. Blue line represents regression, right panel shows the correlation between these parameters. d Percent change of EPSP slope $30 \mathrm{~min}$ after potentiation versus initial peak $\mathrm{Ca}^{2+}$ response of these synapses. Blue line represents regression, right panel shows the correlation between these parameters. e Percent change of EPSP slope $30 \mathrm{~min}$ after potentiation versus initial fraction of suprathreshold EPSPs of these synapses. Blue line represents regression, right panel shows the correlation between these parameters. $f$ Change of response categories of these synapses. 


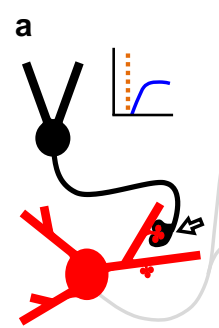

c

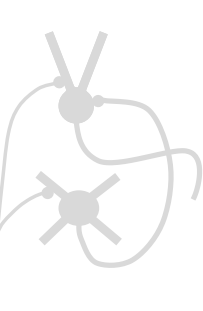

Granule cell

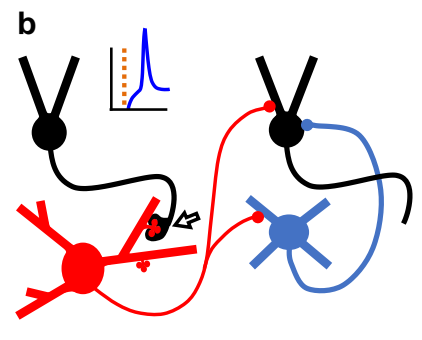

Mossy cell

Stable non-detonator (as in a)
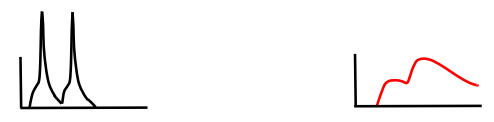

Metastable detonator (mixed responses a and b)

i) AP frequency facilitation or depression
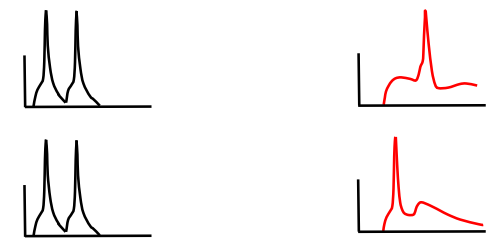

ii) presynaptic EPSP effect
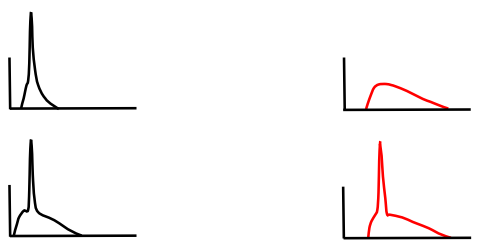

Stable full detonator (as in b)
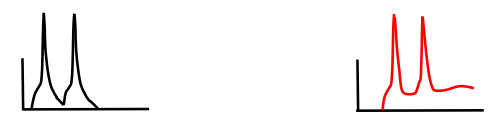

Fig. 6 MF-MC synapses represent a metastable network switch. a If the synapse (open arrow) is in subthreshold mode, only the MC (red) will notice AP firing of the presynaptic granule cell (black). $\mathbf{b}$ In suprathreshold, detonating mode, granule cells and interneurons (blue) postsynaptic to the MC will be notified of this unitary MF-MC EPSP. c MF-MC synapses are encountered in three different states with respect to MC detonation. Stable non-detonator synapses show exclusively subthreshold responses. Metastable detonator synapses are on the edge to detonation. They may become facilitated or depressed and are dependent on AP frequency and accompanying presynaptic EPSPs ${ }^{5656}$. Stable full detonator synapses consistently elicit AP firing of the MC (as illustrated in b). 